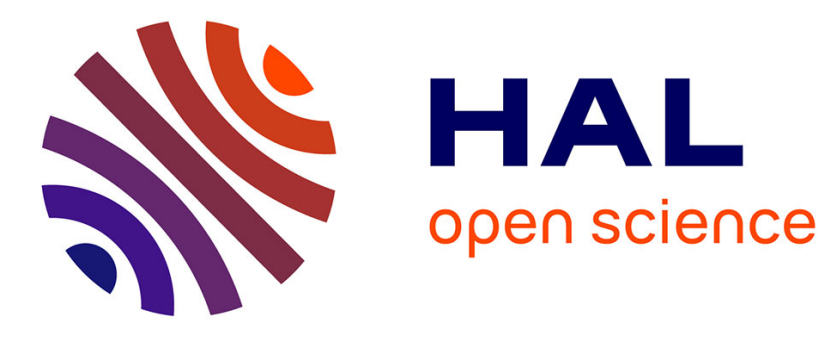

\title{
Dynamic Acousto-Elastic Testing
}

Sylvain Haupert, Guillaume Renaud, Jacques Rivière, Parisa Shokouhi

\section{To cite this version:}

Sylvain Haupert, Guillaume Renaud, Jacques Rivière, Parisa Shokouhi. Dynamic Acousto-Elastic Testing. Nonlinear Ultrasonic and Vibro-Acoustical Techniques for Nondestructive Evaluation, Springer International Publishing, pp.509-546, 2019, 10.1007/978-3-319-94476-0_13 . hal-02989512

\section{HAL Id: hal-02989512 https://hal.science/hal-02989512}

Submitted on 20 Nov 2020

HAL is a multi-disciplinary open access archive for the deposit and dissemination of scientific research documents, whether they are published or not. The documents may come from teaching and research institutions in France or abroad, or from public or private research centers.
L'archive ouverte pluridisciplinaire HAL, est destinée au dépôt et à la diffusion de documents scientifiques de niveau recherche, publiés ou non, émanant des établissements d'enseignement et de recherche français ou étrangers, des laboratoires publics ou privés. 
Chapter 13

Dynamic Acousto-Elastic Testing

\subsection{Introduction}

\subsubsection{Inspirations and Principles of Dynamic Acousto-Elastic Testing

Pioneering measurements of elastic nonlinearity were static methods leading to 7 the thermodynamic diagram that shows the relations between pressure, volume, 8 and temperature (p-v-T diagram) [1]. The dependence of the bulk elastic modulus 9 on the pressure, i.e., a measure of nonlinear elasticity, was deduced from this 10 diagram. In the beginning of the twentieth century, resonance spectroscopy [2, 3] or 11 methods based on interferometry [4] were proposed to measure the elastic moduli 12 as functions of temperature and hydrostatic pressure. Finally, with the possibility 13 of generating an ultrasonic short pulse [5, 6], acousto-elastic testing became an 14 alternative way to assess elastic nonlinearity. Acousto-elastic testing consists in 15 measuring changes of the speed of sound (by the determination of the travel time 16 of an ultrasonic short pulse) induced by a hydrostatic or uniaxial stress (or strain). 17 For metals and polymers, the relative variation in ultrasound wave-speed is found 18

S. Haupert · G. Renaud ( $\triangle)$

Sorbonne Universités, UPMC, CNRS, INSERM, Laboratoire d'Imagerie Biomédicale, Paris, France

e-mail: guillaume.renaud@upmc.fr

J. Riviere

Institut des Sciences de la Terre (ISTerre), CNRS, Grenoble, France

Department of Geosciences, Penn State University, State College, PA, USA

P. Shokouhi

Department of Civil and Environmental Engineering, Penn State University, State College, PA, USA 
between $10^{-5}$ and $10^{-4}$ per MPa of the applied stress. In cracked or granular media, 19 contacts between the two lips of cracks or contacts between grains can greatly 20 increase the variation in ultrasound wave-speed up to about $10^{-2}$ per MPa of applied 21 stress, i.e., orders of magnitude larger than in metals and polymers [7]. 22

A conventional acousto-elastic experiment is quasi-static; the applied stress is 23 varied in discrete steps and the ultrasonic wave-speed is measured for each level 24 of the applied stress [8-10]. While early conventional experiments applied a static 25 stress up to $1 \mathrm{GPa}[6,11]$, recent studies have applied less than $10 \mathrm{MPa}$ [12]. 26 There exist several ways to monitor the change of wave-speed induced by the 27 quasi-static loading. It can rely on an elastic wave (propagating short ultrasonic 28 burst or resonance technique) or on a hybrid optical and ultrasonic approach using 29 diffraction of light by standing elastic wave [13] or Brillouin spectroscopy [14]. 30

In the past three decades, alternative ways to measure the acousto-elastic effect 31 were proposed. A slowly varying sinusoidal loading was proposed instead of a 32 quasi-static stress that is varied in discrete steps [15]. Methods based on the 33 interaction of two bulk elastic waves [16] or surface waves [17, 18] were introduced, 34 including a technique termed Dynamic Acousto-Elastic Testing (DAET) [19, 20]. 35 DAET is the dynamic analog of a conventional experiment of acousto-elasticity, 36 though with significant differences. Firstly, the applied stress is not produced by 37 a universal testing machine in discrete steps but induced by an elastic wave. 38 The elastic wave has typically a frequency of a few $\mathrm{kHz}$ in a lab experiment. 39 Consequently the elastic constants at stake are all adiabatic elastic constants. On 40 the contrary both adiabatic and isothermal elastic constants are involved in a quasi- 41 static conventional acousto-elastic experiment (since a quasi-static deformation 42 is considered to be an isothermal process). Secondly, a low vibrational strain is 43 applied, typically $10^{-6}$, while conventional quasi-static experiments operate with 44 an applied strain exceeding $10^{-4}$. Finally DAET explores the dynamic elastic 45 behavior of a material about its equilibrium state. Unlike conventional quasi-static 46 experiments where a compressive stress only (or tensile stress only) is applied, both 47 tensile and compressive behaviors are investigated in DAET.

In a typical DAET lab experiment, the sample is dynamically excited by an 49 acoustic/elastic wave whose wavelength is larger than the size of the sample 50 (frequency is typically a few $\mathrm{kHz}$ if the sample size is several centimeters). This 51 wave is called the "pump" wave. A sequence of identical ultrasonic short bursts 52 (with a typical center frequency of $1 \mathrm{MHz}$ ) is simultaneously applied to measure the 53 dynamic change of elasticity induced by this pump wave. The ultrasonic short bursts 54 are called the "probe" wave, since they are broadcasted to capture the dynamic 55 variation in material elasticity induced by the pump wave (see Fig. 13.1). The 56 repetition rate of these ultrasonic short bursts is chosen to adequately sample the 57 changes of elasticity produced at a rate imposed by the temporal frequency of the 58 pump wave. Typically the pump wave produces a dynamic acoustic displacement 59 of the order of 1 micrometer while the ultrasonic probe induces a dynamic acoustic 60 displacement of the order of $1 \mathrm{~nm}$. Therefore, the theory of acousto-elasticity as 61 described in [7] can be applied to DAET. 


\section{Author's Proof}

13 Dynamic Acousto-Elastic Testing

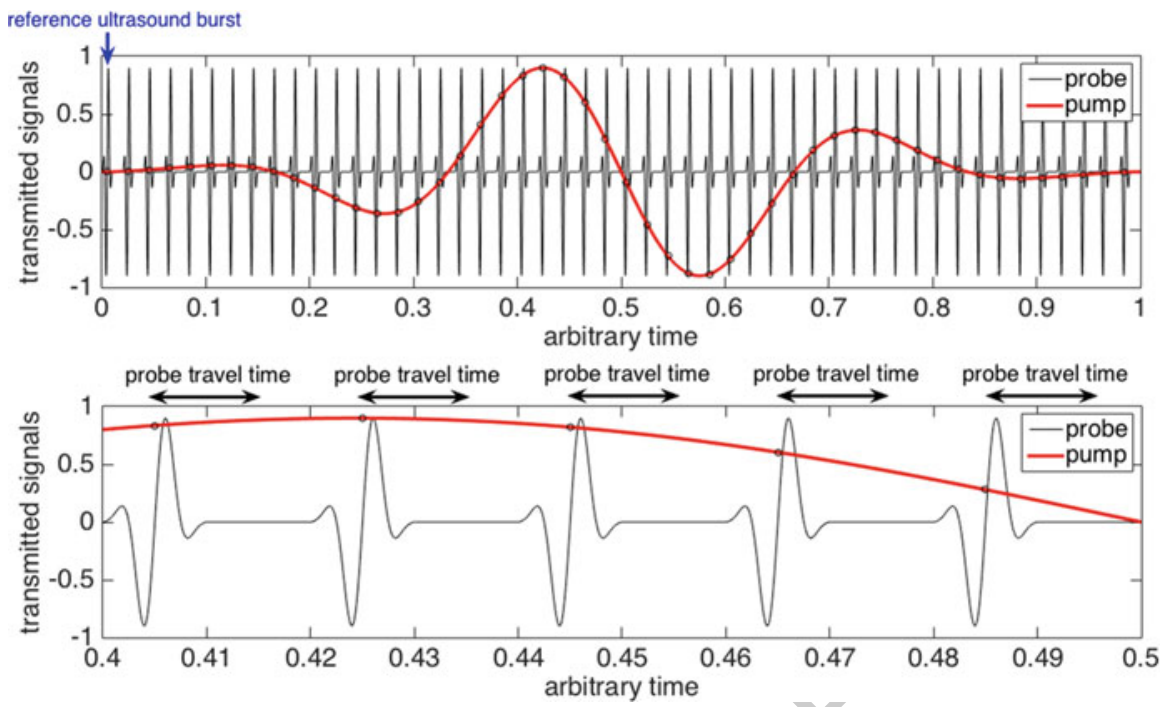

Fig. 13.1 Principles of DAET. Top: schematic representation of the pump wave and the probe wave. The very first ultrasonic pulse is used as a reference. Bottom: enlargement showing that the travel time of an ultrasonic pulse is much smaller than the temporal period of the pump wave

In order to apply the theory of acousto-elasticity [7], the strain field (produced by 63 the pump wave) traversed by the ultrasonic pulses must be quasi-homogeneous and 64 quasi-static with respect to the ultrasonic travel time in the sample. The geometry 65 of the sample and/or the position of the ultrasound transducers that transmit and 66 receive the ultrasonic pulses are then selected so that the travel time of the probe 67 wave (ultrasonic pulses) is much smaller than the temporal period of the pump wave. 68 Each ultrasonic pulse traverses the material as it experiences a different strain level 69 and the large number of ultrasonic pulses in the sequence (typically 1000-10,000). 70 This provides a dense sampling of the relation between the ultrasonic wave-speed 71 measured by the probe wave and the applied strain (produced by the pump wave). 72 The change of ultrasonic wave-speed is calculated from the change of travel time 73 of the probe wave, since the length of the propagation path is known. The change 74 of travel time can be precisely determined from a comparison of a given ultrasonic 75 pulse in the sequence with the very first ultrasonic pulse that serves as a reference 76 (see Fig. 13.1). Techniques to achieve this will be detailed later in this chapter. For 77 each ultrasonic pulse, the strain level is taken as the spatial and temporal average 78 of the strain experienced by the ultrasonic pulse during its propagation through 79 the sample. Techniques to calculate the strain value associated with each ultrasonic 80 pulse will also be explained later in this chapter. 


\subsubsection{Comparison with Other Methods}

DAET belongs to the family of "pump-probe" methods that have existed in 83 nonlinear acoustics from the 1950s [21, 22]. It involves two dynamic fields: one 84 perturbs the material elasticity (the pump) and one measures the induced elastic 85 changes (the probe). Experiments of this type are also termed nonlinear wave 86 mixing. In such measurements, one is interested in the resulting effects of the 87 nonlinear interaction after a propagation distance (i.e., length of interaction) that is 88 much larger than both the pump wavelength and the probe wavelength (see Chaps. 89 1 and 6 in this book). In contrast, in DAET, we are interested in the nonlinear 90 interaction between the pump wave and the probe wave over a distance that is 91 much smaller than the pump wavelength. This situation is therefore more similar 92 to a conventional quasi-static acousto-elastic experiment than an experiment of 93 nonlinear wave mixing.

Nonlinear resonance ultrasound spectroscopy (NRUS, see Chaps. 2 and 12 in 95 this book) measures a variation in the material elasticity (as the driving amplitude 96 is increased) that is time-averaged over an acoustic period. In contrast DAET 97 allows one to "read" the instantaneous variations in the elastic modulus during 98 an entire acoustic cycle of the pump wave. In this respect, DAET provides more 99 detailed insight into the dynamic elastic nonlinearity of a material than NRUS. 100 Moreover nonlinear resonance ultrasound spectroscopy measures the global elastic 101 nonlinearity of the entire sample. In contrast DAET provides a local measurement 102 of elastic nonlinearity. The investigated region of the sample is the volume of the 103 sample that is traversed by the ultrasonic short bursts (probe wave).

\subsection{Experimental Setups}

Dynamic acousto-elastic testing (DAET) was originally developed to evaluate 106 microdamage in trabecular (spongious) bone [19, 23]. In this first configuration, 107 the bone sample was immersed in a water tank. It was later shown that small 108 modifications of this experimental setup allow one to investigate any material 109 immersed in a coupling fluid, such as a plain block of a given solid material, water- 110 saturated glass beads [24], gels or creams [25], or a suspension of particles [25, 111 26]. Meanwhile, DAET method was also extended to experimentations in contact 112 (without the need of immersing the sample in a water tank) to study materials such 113 as rocks [27, 28], concrete [29, 30], or metals with cracks [31, 32] under room-dry 114 conditions. 


\section{Author's Proof}

13 Dynamic Acousto-Elastic Testing

\subsubsection{Low-Frequency Pump Wave: Quasi-Homogeneous and Quasi-Static Requirements}

Whether DAET is done in immersion or in contact, both setups are similar and 118 consist in broadcasting a low-frequency (LF) pump wave and measuring changes 119 of wave-speed experienced by an ultrasonic (US) probe wave that traverses the 120 LF pressure/strain field generated in the probed volume. The analysis of the 121 measurements is straightforward if two requirements are respected: the LF pressure 122 field must be (1) quasi-homogeneous in the volume probed by the US probe wave 123 and (2) quasi-static with regard to the travel time of the US probe wave. In these 124 conditions, it is analogous to conventional quasi-static acousto-elastic testing. 125

It has been shown experimentally and validated by simulation [33] that the 126 pressure/strain field seen by the US probe is quasi-homogeneous when the distance ${ }_{127}$ $d_{\text {probe }}$ traveled by the US probe is at least 10 times smaller than the LF pump 128 wavelength $\lambda_{\text {pump }}$, while the quasi-static requirement is reached when the US time 129 of flight (TOF) propagation is at least 10 times smaller than the LF pump period 130 $T_{\text {pump. }}$. The two requirements are related via the wave-speed $c$ since $d_{\text {probe }}=c^{*}$ TOF 131 and $\lambda_{\text {pump }}=c^{*} T_{\text {pump. }}$. In practice, the LF pump wave is either a standing wave (e.g., 132 first compressional mode of a bar) [27, 34] or a propagative wave [20, 26, 35] (Fig. 133 13.2). Both configurations will be explained in detail in Sects. 13.2.4 and 13.2.5.
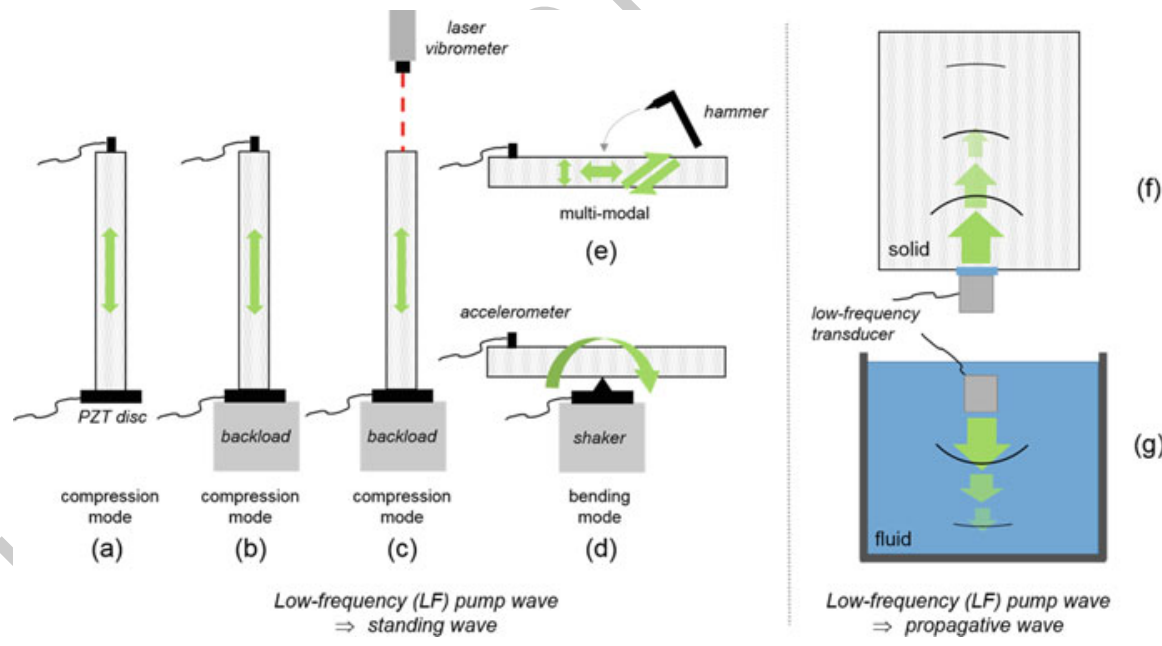

Fig. 13.2 Examples of different low-frequency (LF) pump wave configurations. For bars or cylinders, the LF pump wave can be a standing wave matching either the 1st compressional mode (a-c) or the 1st bending mode (d). The LF pump can also be multi-modal when using a hammer as exciter (e). Various boundary conditions and emitter/receiver types can be used depending on the application. For a (semi-)infinite solid (f) or fluid (g) sample, the pump can be a propagative wave induced by a LF transducer 


\subsubsection{Ultrasonic Probe Wave: Type, Amplitude, Position, and Orientation}

A US probe wave is required to read the instantaneous change of phase velocity 137 at different strain levels. While it is possible to use any kind of ultrasonic waves, 138 bulk compressional wave is involved in most of the studies, while bulk shear wave 139 $[29,36]$, or surface wave such as direct or head wave [27, 37], or Raleigh wave [29, 140 30] remain anecdotic. The only requirement is to be able to measure the TOF of 141 the probe wave and its variation (induced by the pump wave, see Sect. 13.3) along 142 a known propagation path for different strain levels. The propagation path of the 143 probe wave is also needed to evaluate the pump strain experienced by the probe 144 wave.

In practice, this can be done with two US transducers in direct or indirect 146 transmission configuration or with one transducer in pulse-echo configuration (Fig. 147 13.3). The choice of configuration depends on the experimental conditions such 148 as the workable surface of the sample, the material access, the attenuation in the 149 material, or the orientation between the US probe beam and the LF pump wave 150 (e.g., collinear, orthogonal, or with an arbitrary angle). It also depends on how large 151 the TOF variation is along the propagation distance in the medium. If the one way 152 direct transmission path is not enough to accumulate sufficient change in TOF (i.e., 153 larger than the phase noise level), the use of multiple reflections (within the sample) 154 along the same direct transmission path represents a good alternative [32].

In order to determine the propagation path of the US probe wave, a short US pulse 156 is generally preferred as it is relatively easy to guess the propagation knowing the 157 nature of the wave (e.g., compression or shear bulk wave, Rayleigh wave or Lamb 158
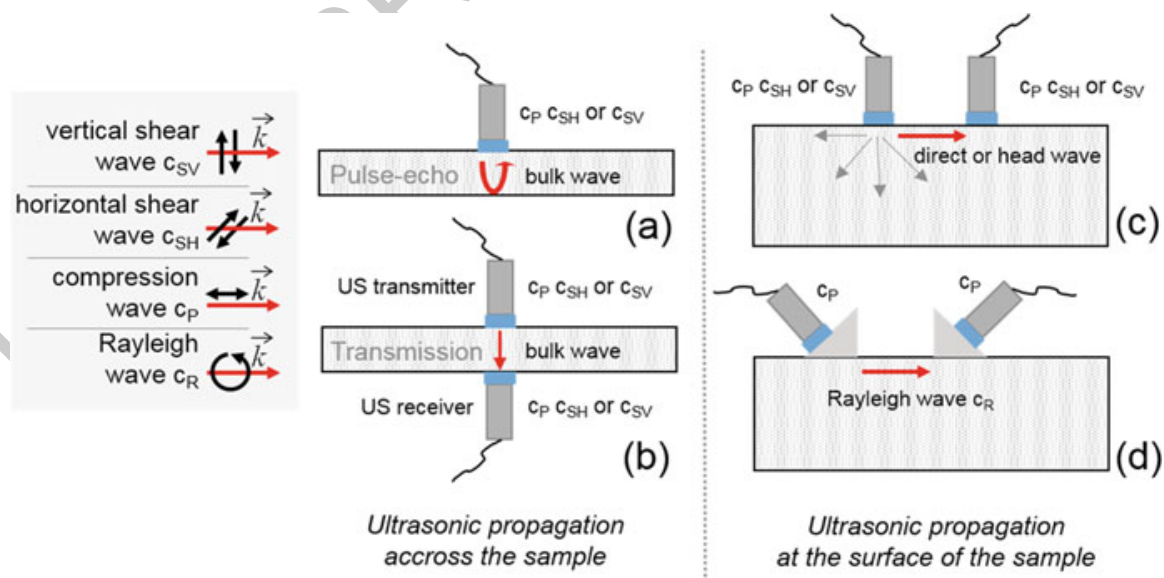

Fig. 13.3 Examples of different high-frequency (HF) probe wave configurations: bulk compression wave or bulk shear wave propagating across the sample in pulse-echo (a) or transmission (b); direct wave (c) or surface wave (d) propagating at the surface of the sample 


\section{Author's Proof}

13 Dynamic Acousto-Elastic Testing

wave). The use of a continuous monochromatic US wave may be used cautiously 159 as waves may travel in the whole material, with possible multiple reflections and 160 modes conversion. The probe signal recorded at the receiver contains the direct 161 propagation signal superimposed with signals produced by reflections and mode 162 conversion within the sample, which makes the analysis difficult. It is preferable to 163 use a continuous probe in case of strong attenuation (e.g., due to multiple scattering 164 and/or absorption) that reduces the amplitude of waves reflected at the sample 165 boundaries [38].

For very heterogeneous media such as rocks or concrete, the direct or ballistic 167 US wave is sometimes difficult to disentangle from the later arriving waves, the 168 so-called coda. The coda is the result of multiple scattering that occurs when the 169 US wavelength is close to the size of the scatterers and/or when the scattering 170 efficiency is high (large contrast of mass density and/or compressibility). A coda- 171 based technique called Coda Wave Interferometry (CWI) can be used to calculate 172 small variations in wave-speed with a greater accuracy than ballistic wave arrival 173 times, making it an interesting tool for measuring acousto-elastic effects [39, 40]. 174 The main drawbacks of using coda wave instead of direct wave are (1) the LF strain 175 field seen by the coda wave is no more quasi-homogeneous nor quasi-static and (2) 176 the coda wave is a superposition of multiple shear and bulk waves having different 177 speed of sound and polarization.

In the case of a porous medium like rocks, it is important to add treatment on the 179 surface of the sample (e.g., polished nail or tape) to prevent coupling ultrasound gel 180 from penetrating the material.

When a short US pulse is used, the pulse repetition frequency (PRF) is 182 conditioned by the distance between the US emitter and receiver and by ultrasound 183 attenuation. Indeed, for proper analysis, two successive US signals (including direct 184 propagation, (multiple) reflections, guided propagation, and (multiple) scattering) 185 must not overlap in the time domain. The ratio between the PRF and the frequency of 186 the LF pump wave must not equal a rational number in order to create a stroboscopic 187 effect, so that US pulses probe different LF strains during each LF cycle. In this 188 manner, US pulses are able to probe discrete values well distributed over the entire 189 LF strain excursion, both in tension and in compression, after several LF periods 190 (e.g., generally between few tens to few hundreds).

\subsubsection{Clock Synchronization and Phase Noise}

The DAET measurement protocol involves three distinct phases (Figs. 13.1 and 193 13.14):

- Pre-pump reference phase-The US probe is turned on, and stays on until the 195 end of the DAET measurement. During this phase, the reference TOF is measured 196 while the noise floor (e.g., phase noise) is evaluated by computing the phase-shift 197 between US signals (i.e., US pulses or US monochromatic continuous wave). 
- Pump phase-The LF pump wave is then turned on. It can be an impact or 199 continuous monochromatic wave, which lasts as long as it is needed for the US 200 signal to probe the entire LF strain excursion. In case of standing wave, it is 201 important to wait for the steady state. The TOF variation is evaluated by assessing 202 the phase-shift between the instantaneous US signal and the reference US signal 203 (see Sect. 13.3)

- Post-pump reference phase or recovery phase-The LF pump wave is finally 205 turned off. In some cases, the phase velocity of the material does not return back 206 instantaneously to its initial value, leading to a relaxation period, due to the so- 207 called conditioning (see Sect. 13.4.2.3) that is tracked by the US probe.

The key for effective DAET measurements is to achieve high sensitivity to 209 small TOF variations. For weakly nonlinear elastic materials, such as PMMA or 210 duralumin, the maximum TOF variation is close to one nanosecond for a US wave 211 traveling through a few centimeters if the maximum LF strain level is in the order of 212 $10^{-5}[27,41]$. Although not straightforward, it is possible to achieve phase noise 213 as low as $0.1 \mathrm{~ns}$ with conventional electronic devices (e.g., function generator, 214 digitizer) if some essential rules are respected.

The most important rule is to maximize the signal-to-noise ratio of the recorded 216 probe signal. Another important aspect is synchronization. All the electronic 217 devices involved in the experimental setup must be synchronized by choosing a 218 single master clock across all devices. This is generally done by connecting the 219 $10 \mathrm{MHz}$ reference clock from one of the devices (e.g., a function generator) to 220 other electronic devices (e.g., the other function generators and digitizers). A good 221 synchronization reduces drastically the electronic time deviation or phase-shift, also 222 called jitter, which is one of the major sources of phase noise. 223

Phase noise can also be caused by relative movements of both US transducers due 224 to low vibrations coming from the environment (e.g., vibration from the building). 225 The use of the same holder for both US transducers overcomes this problem by 226 suppressing the relative movements.

A good configuration for the digitizer (i.e., sampling frequency and quantization 228 bits) is also required to achieve high sensitivity to small TOF variation. High sam- 229 pling frequency is a necessity, but it does not determine directly the TOF resolution. 230 Indeed, TOF resolution depends on the sampling frequency (i.e., sampling period) 231 as well as the numerical tool used to compute the phase-shift between two probe 232 signals (see Sect. 13.3).

The quantization bits (i.e., the number of vertical bits used for analog-to-digital 234 conversion) are generally forgotten but this factor is as important as the sampling 235 frequency. Indeed, the higher the number of quantization bits, the better will be the 236 TOF resolution. The vertical range (i.e., in voltage) of the digitizer must also be 237 adapted to the voltage of the US transmitted signal in order to reach at least $80 \%$ of 238 the full range.

In practice, for short US pulses centered at around $2 \mathrm{MHz}$, a sampling frequency 240 above $50 \mathrm{MHz}$ (i.e., a sampling period of $20 \mathrm{~ns}$ ) with a quantization above 14 bits 241 reduces the phase noise below $0.1 \mathrm{~ns}$. 


\section{Author's Proof}

13 Dynamic Acousto-Elastic Testing

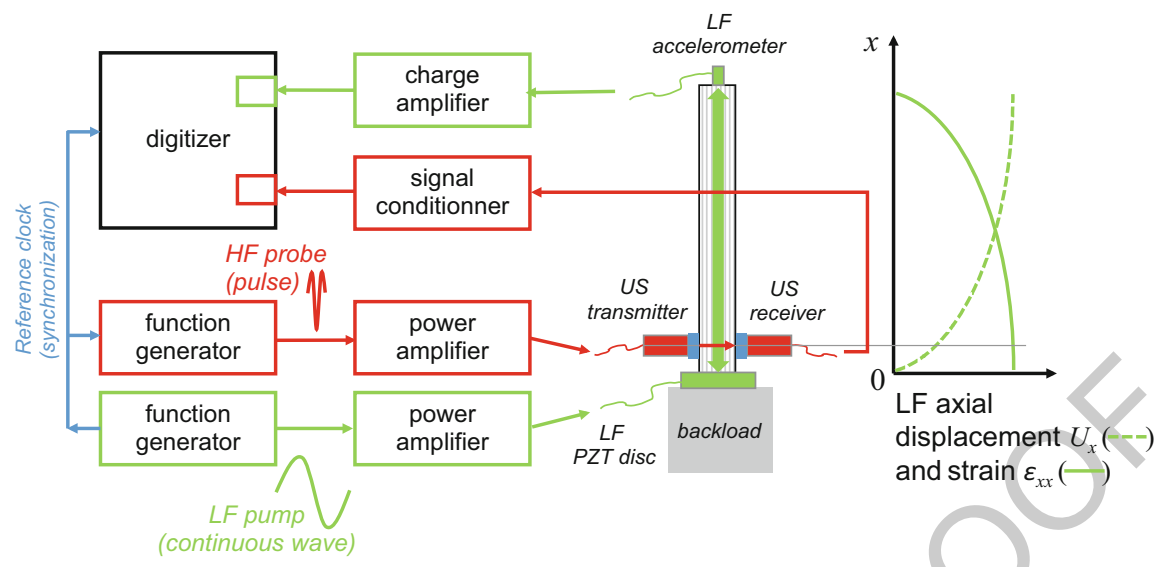

Fig. 13.4 Example of a DAET experimental setup with stationary wave (1st compression mode) as pump wave. The shape of the sample is either a bar or a cylinder

\subsubsection{DAET with Stationary Pump Wave}

DAET with stationary pump wave is recommended for laboratory measurements on 244 calibrated samples such as bars, cylinders, or plates because it is possible: $\quad 245$

(1) To achieve high strain level, up to $10^{-4}$.

(2) To estimate the strain level along the US probe path as the strain distribution is 247 known for a simple modal shape.

In practice, the first compressional [27] or flexural [30] mode is preferentially 249 excited with a continuous monochromatic source tuned to match the frequency of 250 the desired mode. The LF pump wave is broadcasted directly in the sample by means 251 of a piezoelectric disk glued on the sample [27] or a shaker attached to the sample 252 [30]. The frequency of the pump wave is chosen to match the fixed-free [27] or the 253 free-free [42] boundary conditions (Fig. 13.2). 254

When using the first compressional mode with fixed-free boundary conditions, 255 the most common configuration for the probe consists of two US transducers in 256 through-transmission configuration, with a direction of propagation either normal 257 to the pump stress direction (Fig. 13.4) [43], collinear to the pump stress direction 258 [27], or at an angle [41]. The pair of US transducers is generally placed where the 259 LF strain amplitude is the largest, i.e., close to the piezoelectric disk (Fig. 13.4). $\quad 260$

Use of the first bending mode is more restrictive because the strain field across 261 the sample is not uniformed. Nonetheless, along a short portion of the sample, the 262 strain field can be considered quasi-uniform when the penetration depth (i.e., one 263 wavelength) of the US beam is smaller than one tenth of the sample thickness. 264 For this reason, only HF surface waves such as Rayleigh wave [30] or head wave 265 may be used. In this configuration, the US probe is primarily sensitive to the strain 266 component parallel to the US wave propagation direction. 
For in situ measurements, when it is not possible/practical to glue or attach a 268 LF source on the structure, a solution is the use of impact source, such as hammer 269 (Fig. 13.2e). Indeed, impacting briefly the surface of the structure may select the 270 resonant modes with the most excitability. The quick change of elasticity following 271 the impact is either probed with short US bursts [29] or a continuous monochromatic 272 US wave [38]. The main drawback is the difficulty to characterize the strain field 273 seen by the US probe as multiple resonance modes are simultaneously excited. 274

Finally, the measurement of the in-plane or out-of-plane vibration of the sam- 275 ple is performed by either an accelerometer [27] or a laser vibrometer [34] 276 (Fig. 13.2a-d). The strain is then derived directly from the particle's displace- 277 ment/velocity/acceleration by analytical derivation [27]. Sometimes, when the 278 boundary conditions are more complex, a numerical simulation is performed to 279 compute the strain based on the experimentally measured out-of-plane or in-plane 280 particle's displacement/velocity/acceleration [44].

\subsubsection{DAET with Propagative Pump Wave}

In case of in situ measurements (e.g., in soil, large concrete structures or water 283 tank), propagating pump wave is generally the only option as no standing wave 284 is feasible. DAET with propagative pump wave consists of a LF pressure wave 285 that is broadcasted in an infinite medium, i.e., reflections at the boundaries of the 286 medium are negligible (Fig. 13.5). DAET investigates the volume corresponding to 287 the volume of interaction of the two acoustic beams (the probe beam and the pump 288 beam).

When the infinite medium is a fluid, e.g., water, the LF pump wave can be 290 generated by a circular piston attached to a shaker [19] or by a LF immersion 291 transducer [26]. The LF hydrostatic pressure is measured with a hydrophone placed 292 close to the probed volume, in order to evaluate the local pressure. Very different 293 materials could be positioned at the volume of interaction, including solids (e.g., 294 trabecular bone, beads), another fluid (e.g., gel or cream), or micro-particles (e.g., 295 ultrasonic contrast agent).

When the infinite medium is a solid, e.g., soil, rock, or large concrete structure, 297 the LF pump wave is generated at the accessible surface of the structure. In case of 298 measurements in soil, the LF source can be the common LF source for underground 299 prospection, such as a mobile hydraulic shaker [45]. The LF strain is deduced from 300 two accelerometers buried in the soil at different depths [45]. These accelerometers 301 are also used to measure the probe wave. 302

If one wants to reproduce propagation of seismic waves in the laboratory, a 303 LF shear or compressional wave can be propagated in a rock [35, 46] while the 304 LF velocity is measured by a laser vibrometer at the surface of the volume of 305 interaction. 


\section{Author's Proof}

13 Dynamic Acousto-Elastic Testing

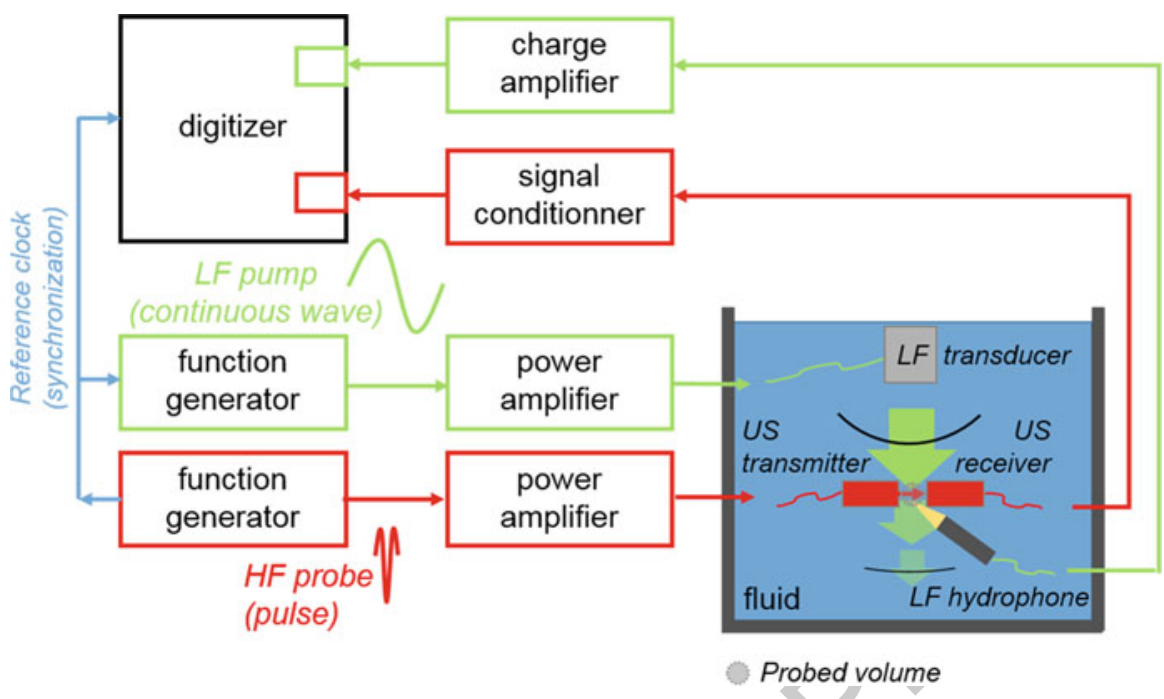

Fig. 13.5 Example of a DAET experimental setup with propagative wave as pump wave. The probed volume corresponds to the volume of interaction of the US beam and the LF beam. The medium is either a fluid or a solid

\subsection{Signal Analysis}

\subsubsection{Analysis of the Pump: Calculation of Strain/Stress Produced by the Pump Wave That is Experienced by the Probe Wave}

Most often, it is desired to know the absolute amplitude of the stress or strain 311 generated by the pump wave. If known, it is possible to compare the elastic 312 nonlinearity of different materials or to measure the third-order elastic constants 313 of a material. Nonetheless, if the objective is to monitor relative changes in elastic 314 nonlinearity of a sample over time (for instance, as a consequence of mechanical or 315 thermal fatigue), the absolute amplitude of the pump wave is not required. In this 316 case a non-calibrated transducer can be used to record the pump wave.

In general it is best to record the pump wave as close as possible to the path 318 of the probe wave in the sample. In a water-borne DAET experiment, the pump 319 wave is measured with a hydrophone, next to the sample immersed in a water tank 320 (Fig. 13.5). In a dry DAET experiment, the pump wave is measured either with 321 an accelerometer or with a laser interferometer or laser Doppler vibrometer (Fig. 322 13.2). However, the recording of the pump wave is often conducted at a position 323 next to the path followed by the probe wave. If the absolute amplitude of the 324 stress or strain generated by the pump wave is required, we need to determine 325 the absolute amplitude of the pump wave experienced by the probe wave along its 326 
path in the sample. Consequently it is necessary to take into account the differences 327 of amplitude and phase between the recording position of the pump wave and the 328 region traversed by the probe wave. To correct these discrepancies, it is usually 329 necessary to measure or calculate the strain/stress field produced by the pump wave. 330

Moreover, the strain field produced by the pump wave is not perfectly uniform. 331 Therefore, the strain/stress level experienced by the probe wave is taken to be the 332 spatial average of the actual strain/stress field produced by the pump wave. Finally, 333 even if the travel time of the probe wave is much smaller than the temporal period of 334 the pump wave, there exists a slight temporal change of the pump amplitude during 335 the travel time of the probe wave. Thus, for an accurate determination of the absolute 336 amplitude of the pump wave, the strain/stress level experienced by the probe wave 337 is taken to be the spatial and temporal average of the pump wave over the region 338 traversed by the probe and during its travel time, respectively.

\subsubsection{Analysis of the Probe: Determination of the Change of Travel Time of the Probe Wave}

The objective of an experiment of acousto-elasticity is to measure the stress- 342 dependence of the speed of sound. Since the distance of the propagation of the 343 probe wave in the sample is known, one must determine the change of travel time 344 of the probe wave induced by the pump wave. There are two ways to determine the 345 change of travel time:

1. The cross-correlation method

2. The phase analysis in the frequency domain

The cross-correlation method consists in computing the cross-correlation func- 349 tion between a reference probe signal (the very first ultrasonic pulse of the sequence) 350 and a second signal that is assumed to be the same waveform as the reference signal 351 with a certain time lag. The time lag, i.e., the change of travel time, is determined 352 by searching the time position of the maximum of the cross-correlation function 353 (Fig. 13.6). It can be positive or negative. Any difference in the waveform that is 354 not a simple difference of amplitude may cause a bias in the determination of the 355 change of travel time. Furthermore a parabolic interpolation around the maximum 356 of the cross-correlation function provides sub-sample estimation of the time delay 357 (i.e., the change of travel time). This technique is widely used in medical ultrasound 358 and is known to be very robust against noise [47]. With an excellent signal-to-noise 359 ratio and an ultrasound frequency of $1 \mathrm{MHz}$, this technique is able to determine a 360 time delay smaller than $0.1 \mathrm{~ns}$.

Another way to determine a change of travel time is the phase analysis in the 362 Fourier domain. At a given frequency $f$ (within the frequency bandwidth of the 363 probe wave), a phase lag $\Delta \varphi$ is simply related to a time lag $\Delta t$ by $\Delta \varphi=2 \pi f \Delta t$. 364 After computing the Fourier transform of the reference probe signal (the very first 365 


\section{Author's Proof}

13 Dynamic Acousto-Elastic Testing
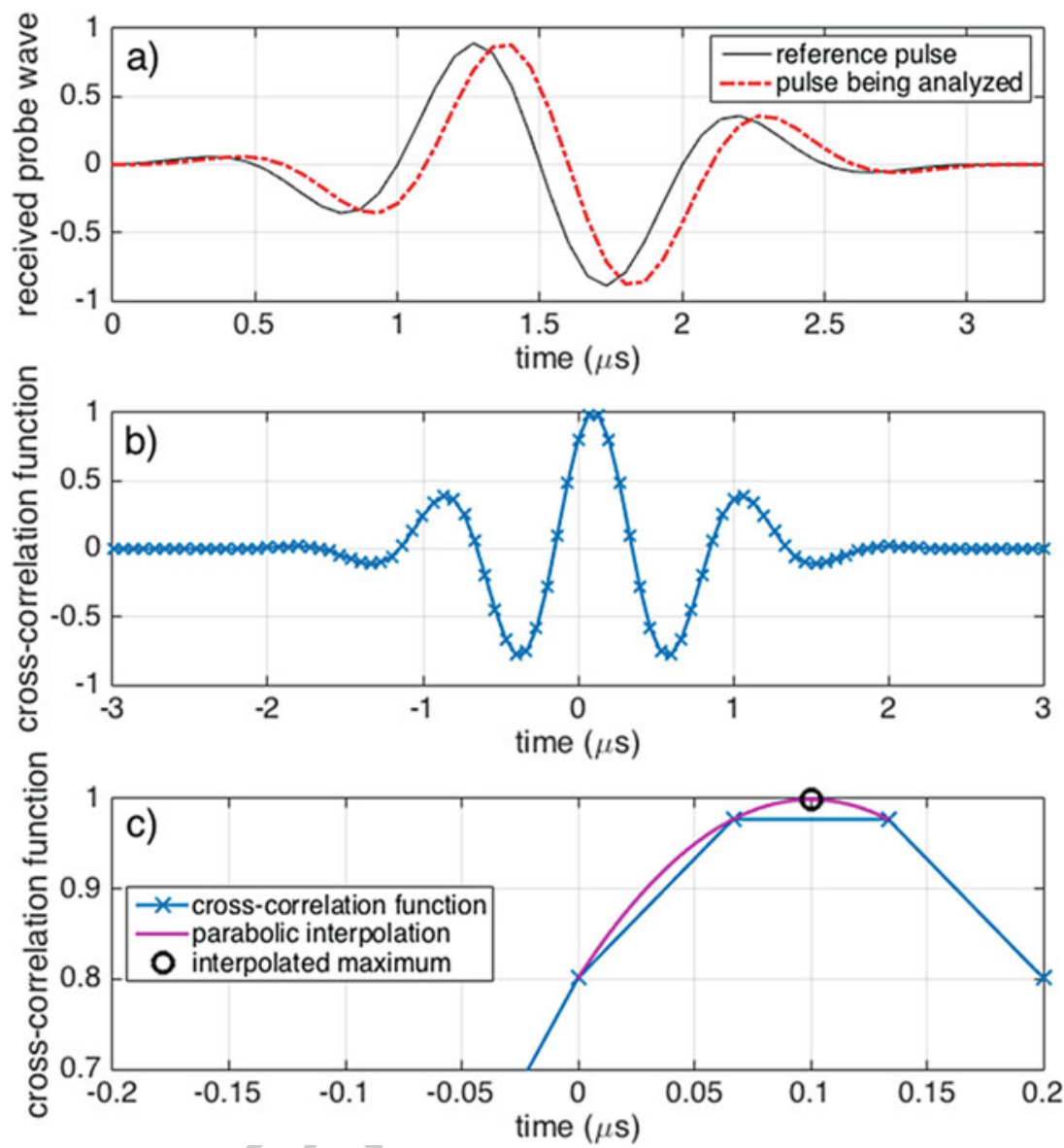

Fig. 13.6 Illustration of the cross-correlation method with synthetic signals. The ultrasonic pulse (probe wave) has a center frequency of $1 \mathrm{MHz}$ and the sampling frequency is $25 \mathrm{MHz}$. (a) Recorded reference signal and signal to be analyzed, a time lag of $0.1 \mu$ s was introduced. (b) Cross-correlation function. (c) Enlargement of panel (b) showing the sub-sample determination of the time lag using a parabolic interpolation of the maximum of the cross-correlation function

ultrasonic pulse of the sequence) and the Fourier transform of one of the following 366 ultrasonic pulses, the phase of the ratio of the two Fourier transforms gives the 367 phase lag (Fig. 13.7). The time lag is then readily deduced. This method is less 368 robust against noise than the cross-correlation technique, but it does not require 369 that the two signals are identical in shape. Besides the phase method is sometimes 370 necessary in a material where ultrasonic attenuation is significantly modified by the 371 pump wave (see later in this chapter). 372

Once the change of travel time of the probe $(d t)$ is accurately determined, the 373 change of wave-speed of the probe $(d c)$ can be calculated after evaluating the change 374 in propagation distance of the probe $(d L)$ induced by the pump strain: 


\section{Author's Proof}

S. Haupert et al.
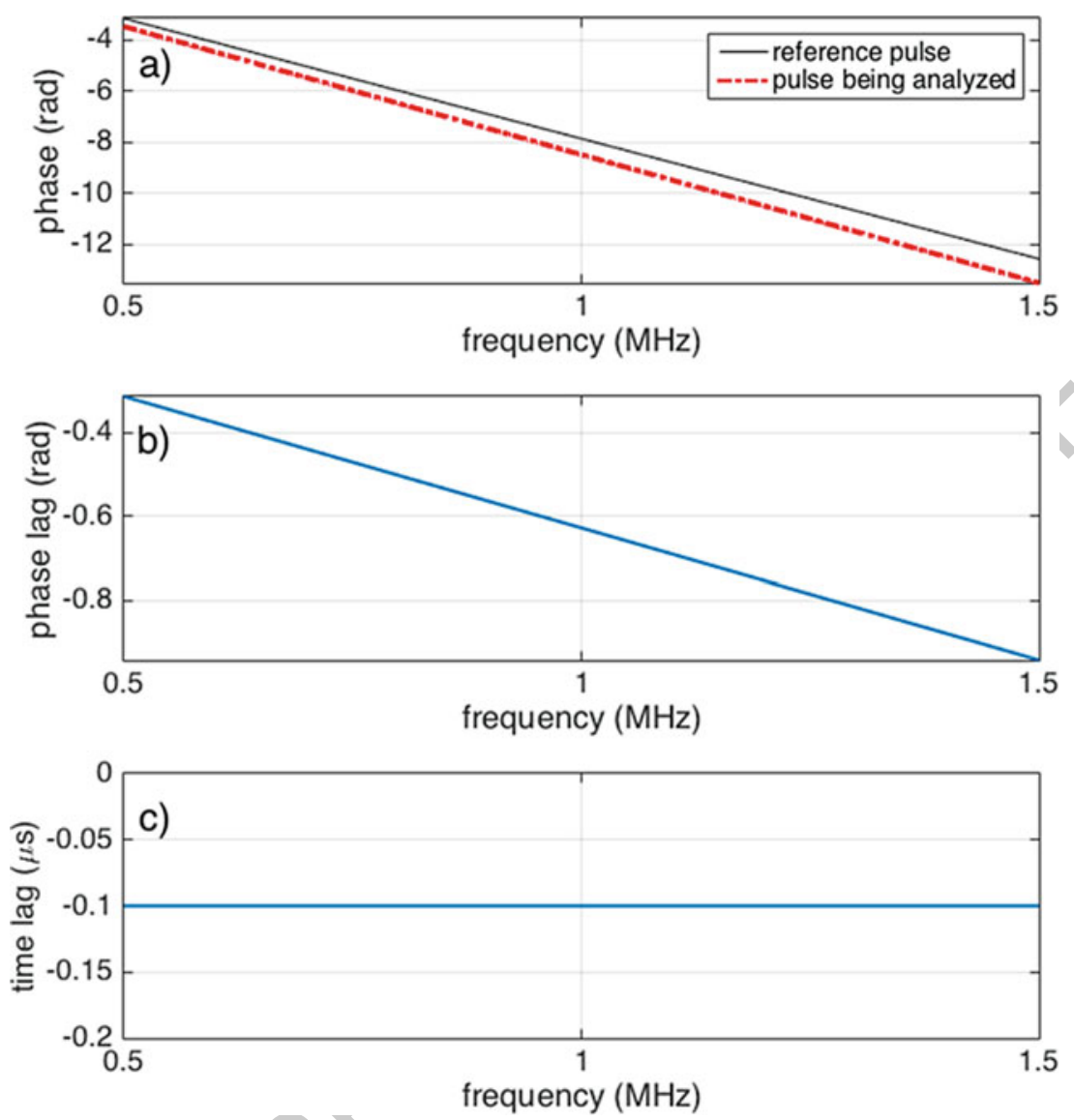

Fig. 13.7 Illustration of the phase method with the same synthetic signals as in Fig. 13.6. (a) Unwrapped phase of the two ultrasonic pulses. (b) Phase lag between the two signals. (c) Calculated time lag

$$
\frac{d c}{c_{0}}=\frac{d L}{L_{0}}-\frac{d t}{t_{0}}
$$

The subscript 0 refers to the value at zero pump strain (at equilibrium, in the 376 absence of pump wave).

If the ultrasonic transducers employed to broadcast the probe and record it are 378 attached to the sample, then $d L$ is readily estimated knowing $L_{0}$ and the strain field 379 produced by the pump wave.

If the ultrasonic transducers are coupled to the sample with water or gel, then the 381 distance between the two transducers is fixed (not affected by the pump). And the 382 formula above must be refined to take into account the variation in travel time in the 383 coupling layer since the thickness of this coupling layer is dynamically modulated 384 by the pump wave. 


\section{Author's Proof}

13 Dynamic Acousto-Elastic Testing

Note also that in materials with high elastic nonlinearity like granular media 386 (rocks, concrete) the correction of the change of distance of propagation of the probe 387 may be neglected since $\left|\frac{d t}{t_{0}}\right| \gg\left|\frac{d L}{L_{0}}\right|$. Nonetheless, in materials with small elastic 388 nonlinearity like polymers, metals, or non-bubbly fluids, this correction must be 389 considered since $\left|\frac{d t}{t_{0}}\right|$ and $\left|\frac{d L}{L_{0}}\right|$ have the same order of magnitude.

\subsubsection{Investigating the Relation Between the Change of Wave-Speed of the Probe and the Magnitude of the Pump Stress/Strain}

We have explained how to obtain two time signals:

1. The change of travel time of the probe as a function of time (i.e., for each 395 ultrasonic pulse of the sequence)

2. The pump strain experienced by the probe as a function of time (i.e., for each 397 ultrasonic pulse of the sequence)

The magnitude of the change of travel time of the probe generally increases as the 399 magnitude of the pump stress/strain is increased. Therefore, a typical experimental 400 protocol includes repeating the measurement while varying the amplitude of the 401 pump wave. Thus we generally have in hand a set of measurements performed with 402 different pump amplitudes. At this point, one has two options to study the relation 403 between the change of wave-speed of the probe and the magnitude of the pump 404 stress/strain:

1. Plot the peak amplitude of the change of travel time of the probe as a function of 406 the peak amplitude of the pump strain/stress

2. Plot the instantaneous change of travel time of the probe as a function of the 408 instantaneous pump strain/stress.

For the first approach, the peak amplitude of the change of travel time of the 410 probe can be estimated directly in the time domain or in the Fourier domain. If 411 the repetition rate of the probe is more than twice larger than the frequency of the 412 pump wave, then a fast Fourier transform can be computed and the amplitude of the 413 variation evaluated. If the repetition rate of the probe is not sufficient to compute 414 a fast Fourier transform (Nyquist criterion is not respected), then a Gram-Schmidt 415 process can be used to decompose the signal, knowing the frequency of the pump 416 wave [34]. Such an analysis is shown in Figs. 13.16, 13.17, and 13.18. 417

The second option is only possible if the recordings of the pump and the probe 418 signals are properly synchronized. This is the method of choice if one is interested 419 in details of the relation between the change of wave-speed of the probe and the 420 magnitude of the pump stress/strain. Such an analysis is shown in Figs. 13.8, 13.9, 421 13.10, 13.11, 13.12, and 13.13 as well as Fig. 13.15. 

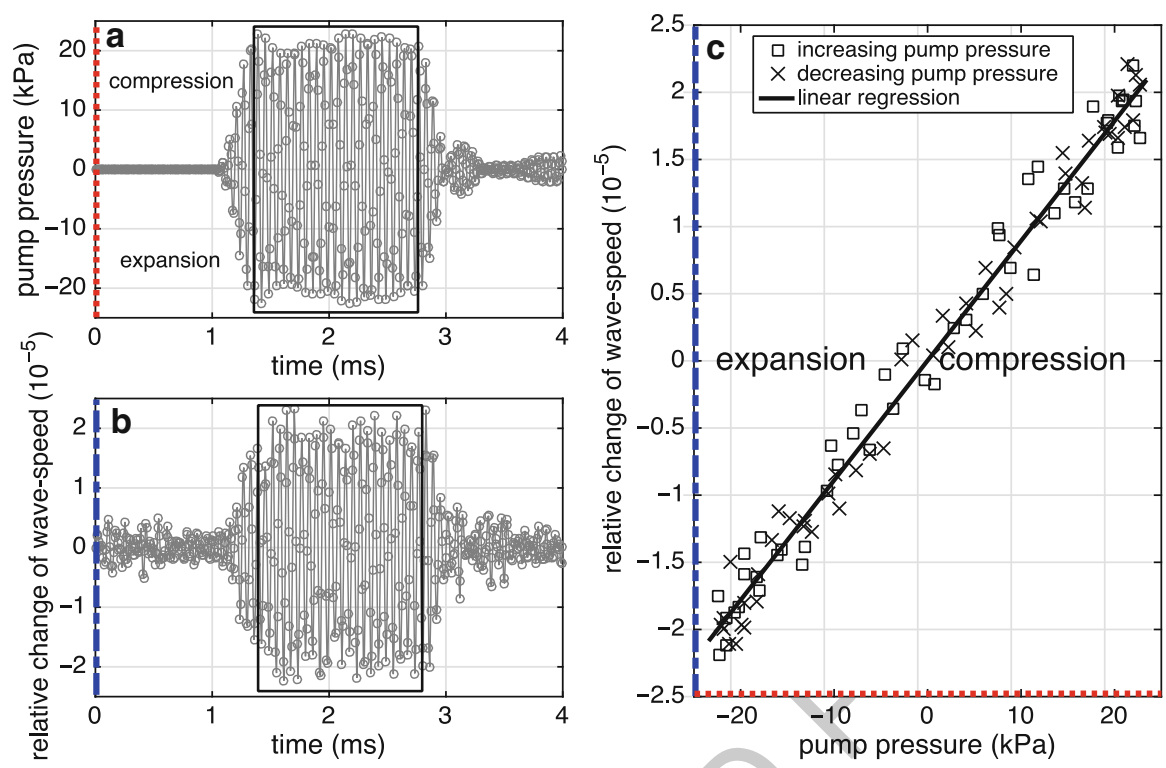

Fig. 13.8 Dynamic acousto-elastic response of water at $20^{\circ} \mathrm{C}$. The experimental setup shown in Fig. 13.5 was used. (a) Pump pressure as a function of time. (b) Relative change of speed of sound as a function of time obtained with the cross-correlation method. (c) Relative change of speed of sound as a function of the pump pressure. The two boxes in panels (a) and (b) show the data points that were selected to plot panel (c)

\subsubsection{Alternative Measures of Acoustic Nonlinearity}

Acoustic nonlinearity includes both elastic nonlinearity and dissipative nonlinearity. 424 In our specific experimental situation, one investigates the effect of a "large- 425 amplitude" pump wave on the propagation of a "small-amplitude" probe wave. The 426 elastic nonlinearity of the material causes the acousto-elastic effect. In addition, 427 in granular media or damaged/cracked media, the pump wave can also induce a 428 variation in the attenuation experienced by the probe wave (dissipative nonlinearity). 429

The objective of a DAET measurement may be very practical, for instance, 430 detecting and monitoring changes in a material like the accumulation of damage. 431 In this case, the investigation of the change of wave-speed of the probe may not 432 be the most sensitive parameter. The change of ultrasonic attenuation may be 433 chosen as an alternative indicator of the level of damage [20, 37]. Such a change 434 of ultrasonic attenuation can be simply implemented by tracking a change of the 435 amplitude of the probe signal. Alternatively the cross-correlation method introduced 436 earlier to estimate a change of travel time of the probe can be further exploited by 437 tracking a change of the amplitude of the normalized cross-correlation function. If 438 its amplitude equals 1 it means that the two input signals are identical waveforms 439 (there may exist a time lag though). If the amplitude of the normalized cross- 440 correlation function is less than 1, it means that the shape of the probe waveform 441 has been modified, likely by a change of ultrasonic attenuation. 


\section{Author's Proof}

13 Dynamic Acousto-Elastic Testing
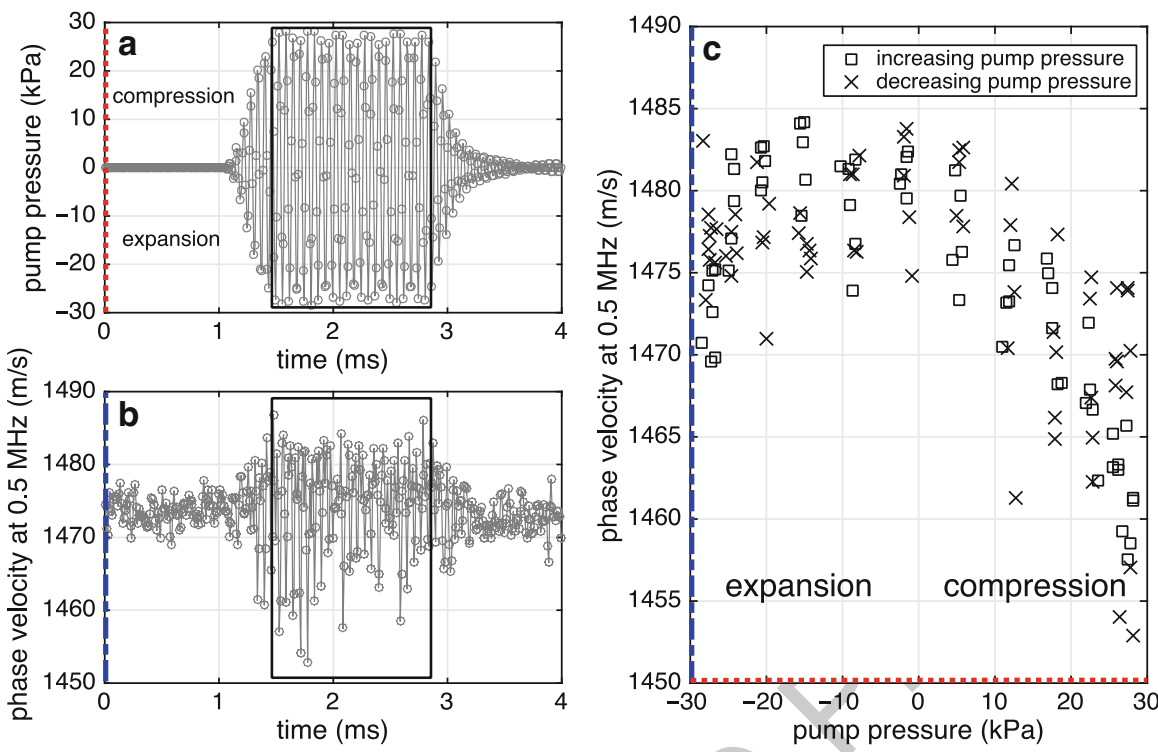

Fig. 13.9 Dynamic acousto-elastic response of a suspension of lipid-coated gas microbubbles in water at $0.5 \mathrm{MHz}$, i.e., at a frequency well below the resonance frequency of the microbubbles. The relative volume fraction occupied by microbubbles is $10^{-6}$. The experimental setup shown in Fig. 13.5 was used. (a) Pump pressure as a function of time. (b) Phase velocity at $0.5 \mathrm{MHz}$ as a function of time obtained with the frequency domain method. (c) Phase velocity at $0.5 \mathrm{MHz}$ as a function of the pump pressure. The two boxes in panels (a) and (b) show the data points that were selected to plot panel (c)
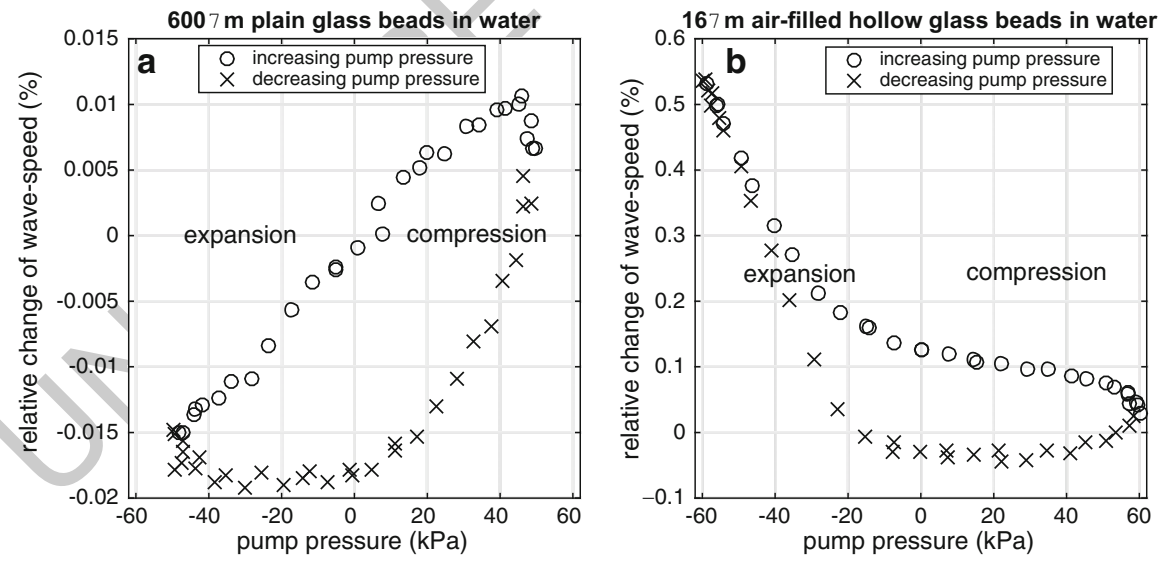

Fig. 13.10 Dynamic acousto-elastic response of (a) water-saturated plain glass beads with a diameter of $600 \mu \mathrm{m}$ and (b) water-saturated air-filled hollow glass beads with a diameter of $16 \mu \mathrm{m}$. These measurements were performed with the system developed by RheaWave [25] which uses the experimental configuration described in Fig. 13.5 


\section{Author's Proof}

S. Haupert et al.

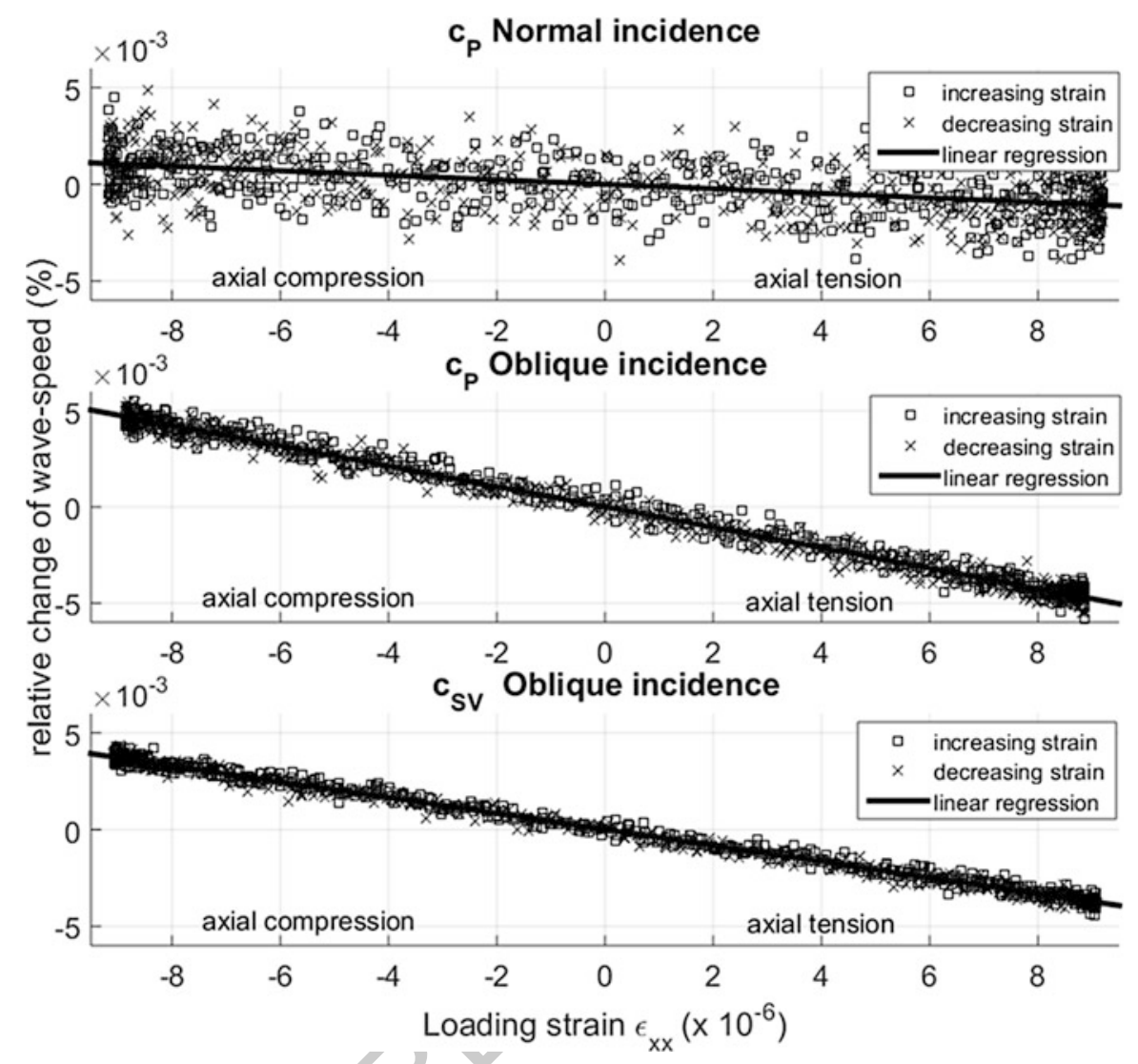

Fig. 13.11 Dynamic acousto-elastic response of PMMA with different orientations and types of US probe waves [41]. The orientations of the ultrasound transducers are chosen to probe the effect of a uniaxial strain (or stress) on the propagation velocity of compression bulk waves $\left(c_{P}\right)$ with normal and oblique incidence and vertically polarized shear bulk waves $\left(c_{\mathrm{SV}}\right)$ with oblique incidence. The maximum axial strain is $9 \times 10^{-6}$ while the relative change of wave-speeds varies between 0.001 and $0.005 \%$

\subsection{Observations in Different Materials}

In this section, we present applications of dynamic acousto-elastic testing to 444 different types of materials. This includes results in liquids and solids. We show 445 how the addition of soft inclusions in a material, namely gas bubbles in a liquid 446 or cracks in a solid, can dramatically change the dynamic acousto-elastic response 447 of the material. More specifically, the reader shall appreciate that the addition of 448 soft inclusions modifies the acousto-elastic effect quantitatively (soft inclusions 449 enhance the elastic nonlinearity of the material) and qualitatively (hysteresis and 450 DC component can appear). 


\section{Author's Proof}

13 Dynamic Acousto-Elastic Testing

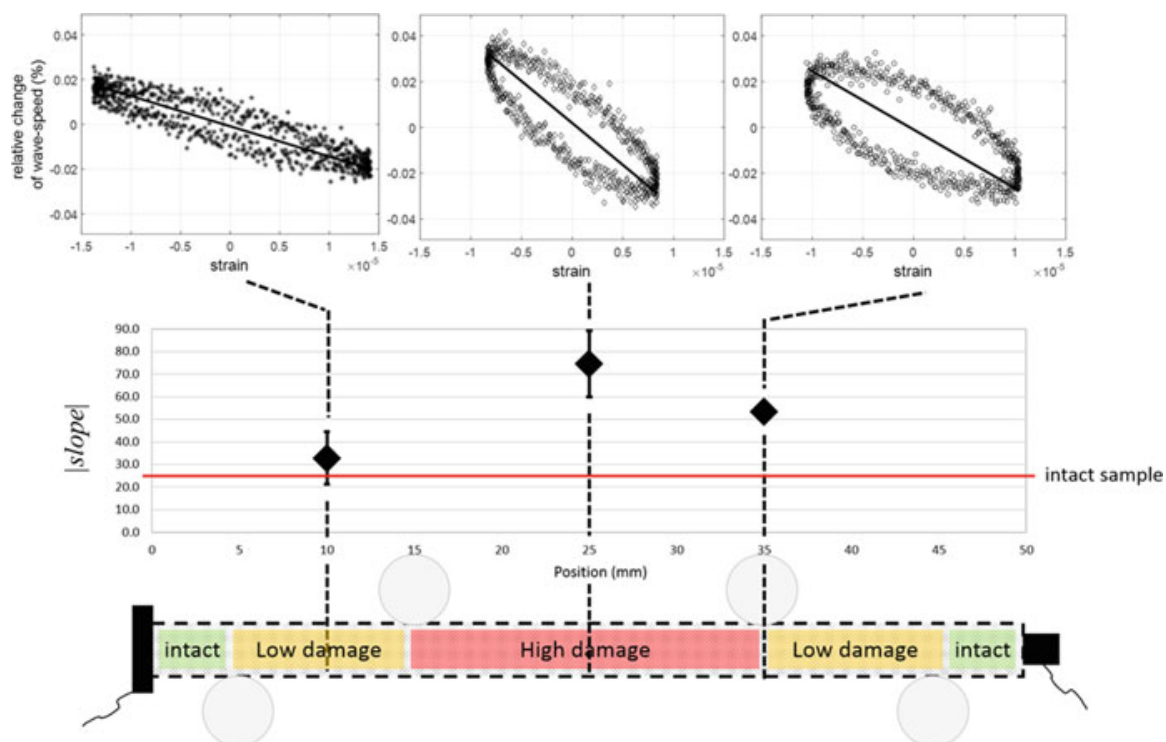

Fig. 13.12 Dynamic acousto-elastic responses measured at different positions along the fatigued bar of aluminum (i.e., 10, 25, and $35 \mathrm{~mm}$ ). The slope and the hysteresis of the nonlinear signature are higher where the level of distributed fatigue damage is expected to be higher. Positive (negative) strain corresponds to tension (compression) phase of the sample

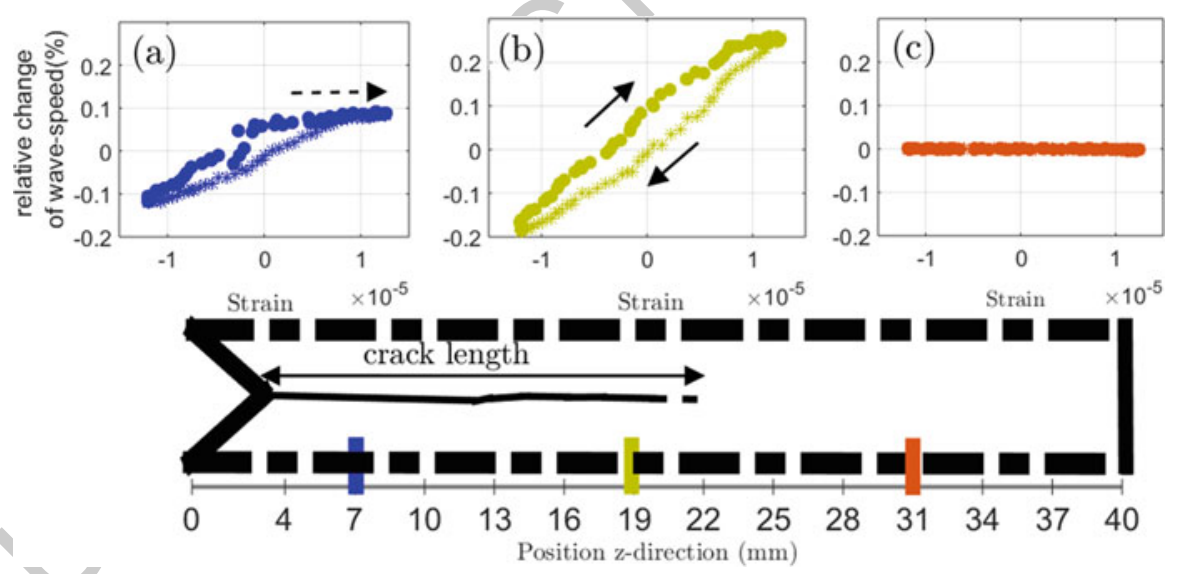

Fig. 13.13 Dynamic acousto-elastic responses for three characteristics positions along the sample width. Positive (negative) strain corresponds to tension (compression) phase of the sample 


\subsubsection{Liquids}

\subsubsection{Non-Bubbly Liquid}

A typical experimental setup to apply dynamic acousto-elastic testing in a fluid is 454 depicted in Fig. 13.5. The pump wave is a $16 \mathrm{kHz}$ pressure wave generated by an 455 underwater acoustic projector. The probe wave is a short ultrasound burst with a 456 center frequency of $4 \mathrm{MHz}$ that is generated by an immersion broadband ultrasound 457 transducer. The ultrasound transducer broadcasts a sequence of short ultrasonic 458 pulses with a repetition rate of $100 \mathrm{kHz}$. A plastic block is used as a reflector 459 placed in front of the ultrasound transducer so that the ultrasound transducer can be 460 operated in pulse-echo mode to transmit and receive the probe wave. The amplitude 461 of the pump wave is first measured by a hydrophone that is inserted between the 462 ultrasound transducer and the reflector, i.e., on the path of the ultrasound pulse 463 (while the probe is turned off). Then the actual experiment of dynamic acousto- 464 elasticity is conducted by turning on both the probe wave and the pump wave. The 465 broadcasting of the pump wave is delayed by $1 \mathrm{~ms}$. This delay is useful to appreciate 466 the level of noise in the measurement of the change of travel time of the probe wave. ${ }_{467}$ The state-of-the-art equipment typically provides a noise level of 0.1 nanosecond or 468 less for the measurement of travel time changes of the probe wave $(d t)$. $\quad 469$

The change of travel time of the probe wave is calculated using the cross- 470 correlation method. Figure 13.8a shows the pump pressure experienced by each 471 probe pulse as a function of time and the panel $\mathrm{b}$ depicts the relative change of 472 speed of sound in water $\left(c-c_{0}\right) / c_{0}$ as a function of time. Finally a plot of the 473 relative change of speed of sound as a function of the pump pressure is shown in 474 panel c. There are several points to highlight when analyzing panel c. Firstly the 475 magnitude of the relative change of wave-speed is small; the change of travel time 476 of the probe wave is close to $0.1 \mathrm{~ns}$ while the total travel time of the probe wave 477 is $5.8 \mu \mathrm{s}$. Secondly panel $\mathrm{c}$ shows that the relation between the relative change of 478 wave-speed and the pump pressure is linear, with no DC offset (the change of wave- 479 speed is null at zero pump pressure) and no hysteresis. When the pump pressure 480 is positive (medium compression) the wave-speed increases. Conversely, when the 481 pump pressure is negative (medium expansion) the wave-speed decreases. $\quad 482$

Finally it was shown that DAET allows one to estimate the parameter of nonlin- 483 earity $\mathrm{B} / \mathrm{A}$ in fluids $[19,25]$. The parameter of nonlinearity $\mathrm{B} / \mathrm{A}$ is proportional to 484 the slope coefficient of the linear relation observed in panel c of Fig. 13.8.

\subsubsection{Liquid with Suspension of Gas MicroBubbles}

The experimental setup depicted in Fig. 13.5 can be used to investigate the influence 487 of the addition of micrometric particles in a fluid. Figure 13.9 shows the acousto- 488 elastic response of a suspension of lipid-coated gas microbubbles in water. Those 489 lipid-coated gas microbubbles are used as an ultrasound contrast agent for medical 490 


\section{Author's Proof}

13 Dynamic Acousto-Elastic Testing

ultrasonography. Even if the microbubbles occupy a relative volume fraction of 491 only $10^{-6}$, the acousto-elastic response of the suspension is dramatically changed 492 compared to that of water only (Fig. 13.8). First the variation in wave-speed is two 493 orders of magnitude larger. Secondly the compression of the medium imposed by 494 the pump wave reduces the wave-speed, while an increase is observed in water only 495 (Fig. 13.8). The suspension of microbubbles has a resonance frequency close to 496 $2 \mathrm{MHz}$. For a frequency well below this resonance frequency, attenuation is very 497 small and the wave-speed is determined by the compressibility of the mixture. 498 Figure 13.9 shows the acousto-elastic response of the suspension at $0.5 \mathrm{MHz}$, it was 499 obtained by applying a frequency domain analysis (see Sect. 13.3 of this chapter). At 500 this frequency, the wave-speed is determined by the compressibility of the medium. 501 The decrease of wave-speed during medium compression is likely caused by the 502 reversible buckling of the lipid shell of the microbubbles [26]. When shell buckling 503 occurs, the stiffness of medium suddenly collapses and the wave-speed decreases. 504

\subsubsection{Water-Saturated Glass Beads}

The acousto-elastic response of water-saturated plain glass beads was investigated 506 $[24,25]$. Figure 13.10 shows the results in water-saturated plain glass beads with 507 a diameter of $600 \mu \mathrm{m}$ and water-saturated air-filled hollow glass beads with a 508 diameter of $16 \mu \mathrm{m}$. The probe signal was a short ultrasound burst with a center 509 frequency of $0.6 \mathrm{MHz}$. The frequency of the pump wave was $3.7 \mathrm{kHz}$. The pump 510 wave is expected to modulate the contacts between the glass beads. As a result 511 complicated dynamic changes of the ultrasonic wave-speed are observed, including 512 expansion-compression asymmetry, hysteresis, and DC offset.

\subsubsection{Solids}

\subsubsection{Undamaged Homogeneous Solids}

Dynamic acousto-elastic testing has been successfully applied to study the clas- 516 sical nonlinear elasticity of two isotropic materials - a polymer, the polymethyl 517 methacrylate (PMMA) and an aluminum alloy [27, 41, 43]. In these studies, both 518 PMMA and aluminum samples have a cylindrical shape; therefore, the LF pump 519 wave is tuned to match the frequency (i.e., few $\mathrm{kHz}$ ) of the first compressional mode 520 (or first Pochhammer-Chree mode) as explained in Sect. 13.2.4. The LF pump wave 521 is broadcasted directly in the sample by means of a piezoelectric disk glued on 522 the sample. The vibration is measured by an accelerometer glued at the top of the 523 sample. The experimental setup of the LF pump corresponds to the configuration 524 shown in Figs. 13.2b and 13.4.

A sequence of ultrasonic short bursts (with a typical center frequency of 1 or 526 $2 \mathrm{MHz}$ ) is simultaneously applied to measure the dynamic change of elasticity 527 
induced by the pump wave. The experimental setup corresponds to the typical 528 configuration shown in Fig. 13.3b but with different angles for the ultrasound 529 transducers that transmit and receive the probe wave. The orientations of the 530 ultrasound transducers are chosen to probe the effect of a uniaxial strain (or stress) 531 on the propagation velocity of compressional bulk waves $\left(c_{P}\right)$ with normal and 532 oblique incidence and with vertically polarized shear bulk waves $\left(c_{\mathrm{SV}}\right)$ with oblique 533 incidence. The maximal pump strain experienced by the US probe is close to $10^{-5}$. 534

The changes in travel time of the probe wave $(d t)$ for different strain levels are 535 calculated using the cross-correlation method. Then, the TOF variation due to the 536 Poisson effect is subtracted from the total TOF variation in order to retrieve the TOF 537 variation solely due to the variation in wave-speed (Sect. 13.3). 538

The results are shown in Fig. 13.11. For undamaged homogeneous solids such as 539 PMMA and aluminum, the relative variation of wave-speed is very weak (between 540 0.001 and $0.005 \%$ ) and exhibits a linear relation with the pump strain. When the 541 pump strain is negative (axial compression), the wave-speed increases. Conversely, 542 when the pump strain is positive (axial tension), the wave-speed decreases. Neither 543 a DC offset nor hysteresis is observed: the change of wave-speed is null when the 544 pump strain is zero.

In particular, a larger variation of compression bulk wave-speed $\left(c_{P}\right)$ is observed 546 when the direction of propagation has an oblique incidence compared with normal 547 incidence. Indeed, it is well established that, even though a homogeneous solid 548 sample exhibits isotropic elastic properties, the effect of a uniaxial strain (or stress) 549 induces wave-speed anisotropy [7]. The propagation velocity of an elastic wave 550 depends on the angle between the propagation direction and the axis of the applied 551 loading [8]. Therefore, the acousto-elastic effect is stronger when the propagation 552 direction of the probe wave is parallel to the direction of the uniaxial loading 553 (produced by the pump wave).

Finally, it has been shown that it is possible to estimate the three independent 555 third-order elastic constants (TOEC) for isotropic materials with DAET, by com- 556 bining three different carefully chosen configurations for the US probe [41].

\subsubsection{Damaged Homogeneous Solids}

In the past decades, several nonlinear acoustical techniques were proposed for in 559 situ nondestructive testing (NDT), such as wave frequency mixing or resonance 560 measurements. Among them, DAET provides a unique way to observe nonlinear 561 elastic features over an entire dynamic stress cycle while other techniques measure 562 average bulk variations of modulus versus strain level. DAET also provides a local 563 measurement of the nonlinear elasticity, which is particularly convenient to localize 564 and characterize microdamage within a whole solid.

It has been demonstrated that DAET applied to steel and aluminum samples is 566 sensitive to 


\section{Author's Proof}

13 Dynamic Acousto-Elastic Testing

1. Distributed fatigue damage [32] and 568

2. A localized single micro-crack $[31,32]$. 569

The experimental setup involves a stationary pump wave and two compressional 570 bulk wave transducers in transmission configuration as described in Fig. 13.4. 571

In case of the fatigue damage protocol [32], two aluminum bars $(50 \times 4 \times 2 \mathrm{~mm}) 572$ are machined from the same $2 \mathrm{~mm}$ thick aluminum plate. The first aluminum 573 specimen is fatigued while the second one is kept intact and serves as control. 574 Fatigue damage is induced by cycling 10,000 times within the elastic regime using 575 a four-point bending configuration. Dislocation density and/or fatigue cracking on 576 the external surface is suspected to be larger in the center of the bar where the stress 577 concentration is the maximum, while both ends of the bar remain intact. DAET 578 measurements have been performed on both aluminum samples (i.e., intact and 579 damaged) at different positions along the bar (i.e., 10, 25, and $35 \mathrm{~mm}$ ). For each 580 position, the relative change of wave-speed is plotted against strain level in Fig. 581 13.12. Then, the slope is extracted from each acousto-elastic response. Negative 582 slopes are observed, as expected for most metals, with the highest value obtained in 583 the center of the specimen, where fatigue damage is expected to be the most severe. 584 Moreover, a hysteresis is present in the nonlinear signature. For the intact sample, 585 no hysteresis is observable and the slopes (horizontal red line in Fig. 13.12) remain 586 constant along the sample with an average value smaller than the slopes measured 587 in the damage sample.

In case of the localized single micro-crack protocol [31], a closed fatigue crack 589 has been formed in an aluminum alloy bar $(170 \times 30 \times 40 \mathrm{~mm})$ by a three-point 590 bending fatigue test. The fatigue crack is extended from a notch placed at mid- 591 length. The notch is approximately $3 \mathrm{~mm}$ deep and the fatigue crack is $17 \mathrm{~mm}$ long. 592 The fatigue crack is invisible to the eye. Twelve DAET measurements have been 593 performed along the crack (i.e., in the z-direction). Three typical acousto-elastic 594 responses are shown in Fig. 13.13. No change in wave-speed is observed outside 595 of the crack (Fig. 13.13c) whereas large changes are observed along the crack (Fig. 596 13.13a, b). The largest slope and hysteresis are observed close to the crack tip (Fig. 597 $13.13 \mathrm{~b})$. At the notch, the crack is expected to be more open than at any other 598 location. A bi-state behavior is clearly observed near the notch (Fig. 13.13a), with 599 the presence of a plateau, i.e., no change in velocity during the tension phase when 600 the crack is opened. Therefore, near the notch, the crack induces elastic nonlinearly 601 essentially during the compression phase while elasticity is virtually unchanged in 602 the tension phase. This agrees with a simple model of Contact Acoustic Nonlinearity 603 $[48,49]$ that describes asymmetry between compression and tension phases. On the 604 other hand, at the crack tip, the change of wave-speed is more symmetric and a high 605 elastic nonlinearity (large slopes in Fig. 13.13b) is observed. Finally the positive 606 sign of the slope is somewhat surprising as it means that the velocity decreases 607 during the compression phase, which would imply that asperities at the interface 608 slow down the direct wave. Further investigation will be needed to understand such 609 behavior. 


\subsubsection{Rocks, Cementitious, and Granular Materials}

Unlike undamaged or single-cracked materials, poorly consolidated media such 612 as rocks and concrete exhibit very large nonlinear behaviors [50]. Perhaps the 613 most striking feature is the appearance of a transient elastic softening, as soon as 614 the medium is subjected to dynamic strains as low as $10^{-7}$ [27, 37]. It is then 615 followed by a $\log (t)$ relaxation back to the original elastic modulus as soon as the 616 dynamic loading is turned off [51]. This elastic softening is also often referred 617 to as "conditioning," or "dynamically induced conditioning" or "DC offset." In 618 some poorly consolidated media, the elastic modulus can therefore be transiently 619 reduced by several percent, depending on the loading amplitude and frequency. 620 Understanding such behavior is critical to better estimate seismic hazard, for 621 instance, for civil structures and buildings. One key step towards the development 622 of robust diagnostic tools is therefore to relate these complex nonlinear responses to 623 physical, quantitative microstructural features.

Beyond nondestructive evaluation and civil engineering applications, there is 625 growing evidence that such nonlinear effects are key mechanisms for the under- 626 standing of earthquake triggering, when a large seismic wave transiently softens the ${ }_{627}$ Earth's crust and triggers a second earthquake [52]. Current research also focuses 628 on the link between elastic softening [53] and transient increases in permeability 629 observed following earthquakes [54]. Such relation with fluid processes is of 630 particular significance for the understanding of induced seismicity in oil/gas and 631 geothermal applications.

As shown in Fig. 13.14, the sound speed evolution in highly nonlinear media 633 such as rock, concrete, and granular media can be decomposed in six consecutive 634 phases.

The initial phase (Phase I) refers to the beginning of the experiment when the 636 pump is "off." In this phase, the unperturbed local wave-speed of the specimen is 637 registered. Upon turning "on" the pump, the pump strain first rings up towards the 638 steady state (Phase II). In Phase III, the pump strain has reached a steady state, but 639 the sound speed continues to drop. Phase IV is the time domain where the medium 640 is in a non-equilibrium steady state. By analyzing the strain-dependency of sound 641 speed in this phase, the different nonlinear material properties can be evaluated, as 642 discussed in the next section (Phase IV: During the Dynamic Loading). In Phase 643 $\mathrm{V}$, the pump strain rings down towards zero. Finally in Phase VI, the pump strain 644 is zero, but the sound speed is still recovering. The slow recovery of sound speed 645 towards the speed of sound at the initial unperturbed state is referred to as "slow 646 dynamics" and will be further detailed in section "After the Dynamic Loading: Slow 647 Dynamics (Phase VI)". 


\section{Author's Proof}

13 Dynamic Acousto-Elastic Testing

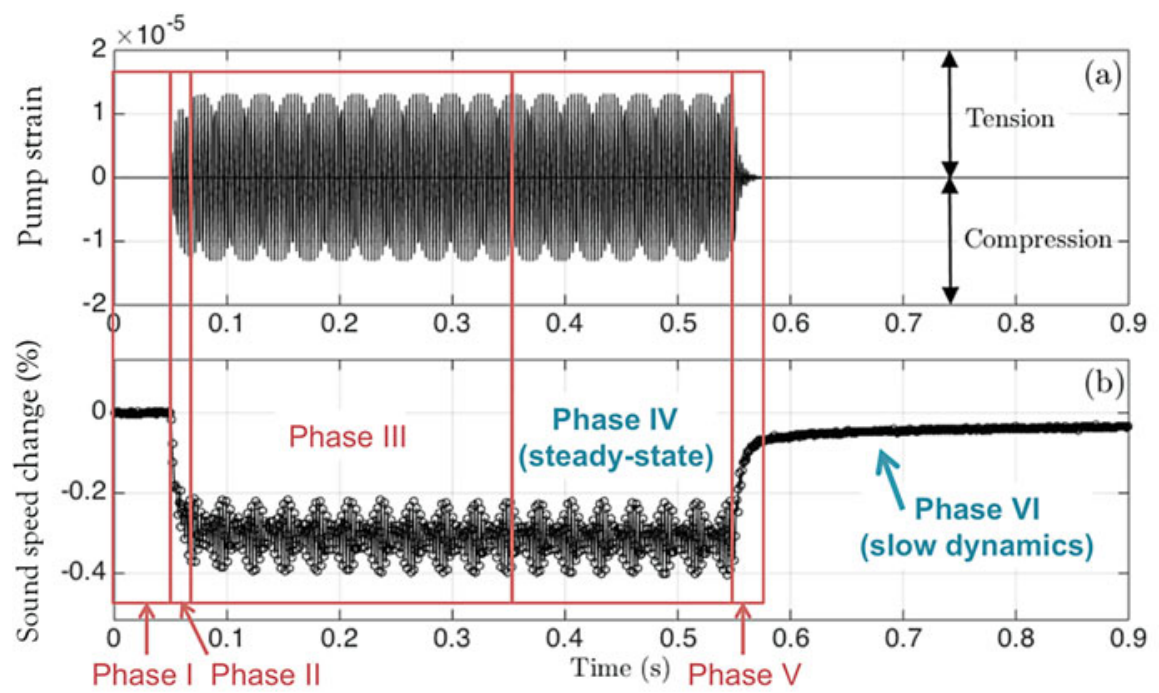

Fig. 13.14 Typical DAET results in a sample of Berea sandstone [28] using the experimental setup described in Fig. 13.4. The sound speed changes in response to the pump strain. The evolution of AQ5 sound speed can be studied in six time domains (Phases I-VI). Details on the fast (Phase IV) and slow (Phase VI) dynamics responses are presented in sections "Phase IV: During the Dynamic Loading" and "After the Dynamic Loading: Slow Dynamics (Phase VI)," respectively

Phase IV: During the Dynamic Loading

DAET is often performed at a single large strain amplitude, either to evaluate the 651 third-order elastic constants in weakly nonlinear elastic materials (Fig. 13.11), to 652 compare the nonlinear responses at different locations (Figs. 13.12 and 13.13), or to 653 monitor a sample over time as it undergoes progressive damage. On the other hand, 654 to further improve the theoretical description of nonlinear elasticity in complex 655 (either damaged or granular-like) materials, one may want to perform DAET at 656 multiple peak strain amplitudes, from a weakly nonlinear regime at low strain 657 $\left(\sim 10^{-7}\right)$ to a highly nonlinear regime at larger strain $\left(\sim 10^{-5}\right)$.

Figure 13.15 shows some typical DAET results in two samples of Berea 659 sandstone and Berkeley blue granite at room-dry conditions and multiple strain 660 amplitudes ranging from $10^{-7}$ to $10^{-5}$, using the experimental setup described in 661 Fig. 13.4. Both samples exhibit a transient elastic softening that increases with 662 the pump amplitude, reaching about $\sim 0.5 \%$ at strain $\varepsilon_{m}=8 \times 10^{-6}$. Further 663 observations can be made depending on the strain range. At large strains $\left(\sim 10^{-5}\right)$, 664 complex loops are observed, with larger (lower) velocity during the compression 665 (tension) phase, suggesting opening/closing of micro-cracks or grain contacts. This 666 effect seems particularly strong for the granite sample (Fig. 13.15b). The acousto- 667 elastic responses for the two samples differ at intermediate strains $\left(\sim 10^{-6}\right)$; while 668 


\section{Author's Proof}

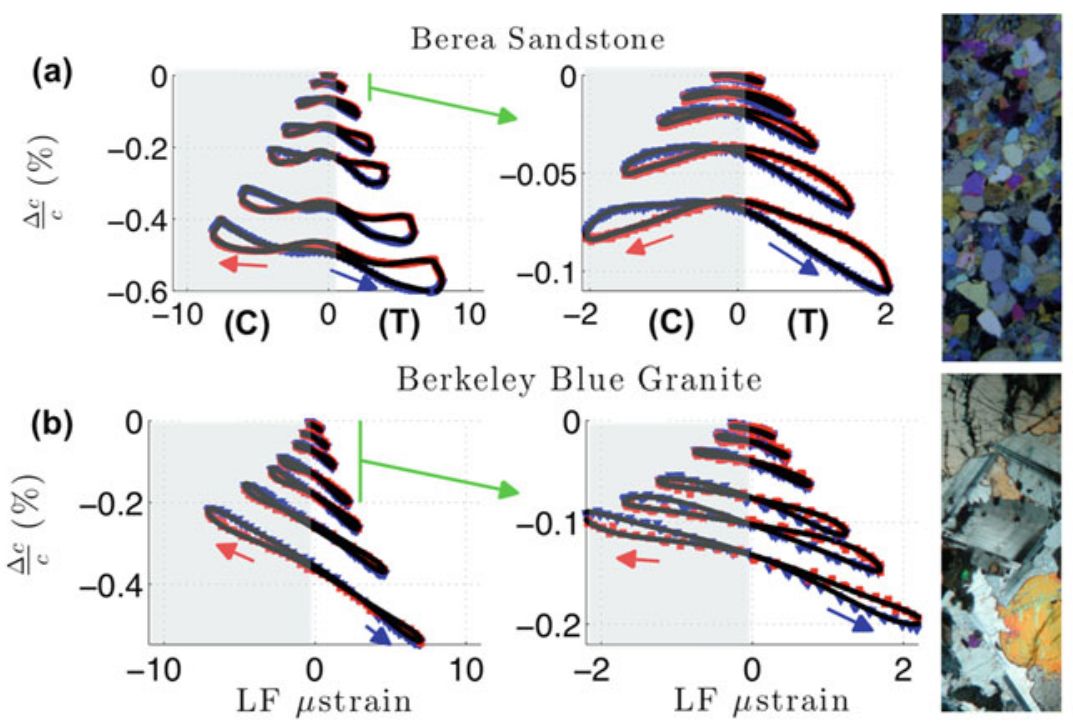

Fig. 13.15 Relative velocity change $\Delta c / c$ as a function of low-frequency pump strain $\varepsilon$ for 10 increasing strain excitations ranging from $10^{-7}$ to $10^{-5}$. (a) Berea sandstone. (b) Berkeley blue granite. Plots on the right side provide details on the nonlinear responses for strain amplitudes lower than $2 \times 10^{-6}$. Blue triangles (red squares) correspond to increasing (decreasing) strains. Negative strains correspond to the compression phase (C) whereas positive strains correspond to the tension phase (T). The black line shows the result of the Fourier analysis (cf. [28] for further details). On the right side, photomicrographs under plane polarized light. Height of the photo is $2.35 \mathrm{~mm}$ for Berea sandstone, $3.85 \mathrm{~mm}$ for Berkeley blue granite (Modified from [28])

the granite sample exhibits a larger velocity at negative pump strain (than at zero 669 pump strain) and a smaller velocity at positive pump strain, the velocity in Berea 670 sandstone at negative and positive pump strain maxima is found lower than at zero 671 pump strain (right plots in Fig. 13.15). No certain evidence exists on a particular 672 mechanism for such observation. However, the fact that it is observed for all tested 673 sandstones [28, 43] suggests that it could arise from shearing processes at the 674 grain boundaries, leading to a lower elastic modulus during maximum tension and 675 compression, and larger moduli when strain passes through zero. Finally, the large 676 slope for granite at intermediate and large strains suggests that the opening/closing 677 mechanism dominates over shearing processes [43].

The dynamic acousto-elastic response of rocks is far more complicated than that 680 of homogeneous undamaged solids (like PMMA, Fig. 13.11). While the velocity in 681 homogeneous undamaged solids is modulated only at the frequency of the pump, 682 the dynamic change of velocity in rocks exhibits a component at the frequency of 683 the pump, at its higher harmonics as well as at zero frequency. To further investigate 684 


\section{Author's Proof}

13 Dynamic Acousto-Elastic Testing

the nonlinear elastic responses in rocks, the time series $\Delta c / c(t)$ can be decomposed 685 using a Fourier analysis in combination with a Gram-Schmidt procedure [34]. The 686 latter is needed to ensure orthogonality of the sine and cosine functions. From this 687 analysis we find the amount of $\Delta c / c(t)$ oscillating at the pump frequency $\Delta c /\left.c\right|_{1 \omega}, 688$ twice the pump frequency $\Delta c /\left.c\right|_{\mathbf{2} \omega}$, as well as the DC offset $\Delta c /\left.c\right|_{\mathbf{0} \omega}$ (zero frequency 689 component). When hysteretic effects are relatively small, as in Fig. 13.15, these 690 quantities correspond approximately to the slope of the signature, the curvature, and 691 the DC offset, respectively.

The $\Delta c /\left.c\right|_{0 \omega}$-component is represented in Fig. 13.16 as a function of pump 693 strain amplitude for both samples. This offset observed with DAET is equivalent 694 to the frequency shift observed with NRUS (see Chap. 2 in this book) [28]. A 695 progressive transition from quadratic dependence at low strain $\left(\sim 10^{-7}\right)$ to linear 696 at large strain $\left(\sim 10^{-5}\right)$ is observed. This result, observed with both DAET [28, 697 34, 37] and NRUS [55], is typical of poorly cemented rocks. While no current 698 theoretical model is able to fully capture the complex nonlinear response of rocks, 699 cementitious, or granular-like materials, it is worth comparing these observations to 700 some existing theories. One practical approach is to use the following 1D-equation 701 (assuming material elastic nonlinearity is large and therefore neglecting changes in 702 mass density):

$$
\frac{\Delta M}{M}=2 \frac{\Delta c}{c}=\beta \varepsilon+\delta \varepsilon^{2}+\alpha\left(\varepsilon_{m}+\operatorname{sign}(\dot{\varepsilon}) \varepsilon\right),
$$

where $\frac{\Delta M}{M}, \frac{\Delta c}{c}, \varepsilon, \dot{\varepsilon}$, and $\varepsilon_{m}$ are, respectively, the relative change in modulus, the 704 relative change in wave-speed, the pump strain, the strain rate, and the maximum 705 strain excursion experienced by the material. The maximum strain excursion $\varepsilon_{m}$ in 706 our case is the amplitude of the pump strain, assuming $\varepsilon=\varepsilon_{m} \sin \omega t$. The terms 707 $\beta$ and $\delta$ represent the nonlinear quadratic and cubic coefficients, respectively, and 708 arise from the classical nonlinear theory [7]. In particular, the term $\beta$ is related 709 to the third-order elastic coefficients (TOEC) [7]. The last term on the right-hand 710 side results from the quadratic hysteretic nonlinear theory [50, 56-58]. Following 711 such description, the quadratic dependence observed at low strain in Fig. 13.16 can 712 be fitted with the cubic nonlinear parameter $\delta$ using $\left.\frac{\Delta \boldsymbol{M}}{M}\right|_{0 \omega}=\left.2 \frac{\Delta \boldsymbol{c}}{c}\right|_{0 \omega}=\frac{\delta \varepsilon_{m}^{2}}{2}, 713$ whereas the linear dependence observed at large strain can be better fitted with the 714 hysteretic parameter $\alpha$ using $\left.\frac{\Delta \boldsymbol{M}}{M}\right|_{0 \omega}=\left.2 \frac{\Delta \boldsymbol{c}}{c}\right|_{0 \omega}=\alpha \varepsilon_{m}$. However, it is important to 715 note that such hysteretic model inherently couples hysteresis with softening which 716 leads to an overestimation of hysteretic effects [34]. It also does not include the 717 rate/frequency/relaxation effects that are described later.

Figure 13.17 shows the $\Delta c /\left.c\right|_{\mathbf{1} \omega}$-component as a function of pump strain 719 amplitude. The linear dependence over the whole strain range can be fitted with 720 the classical quadratic nonlinear parameter $\beta$ using $\left.\frac{\Delta \boldsymbol{M}}{M}\right|_{1 \omega}=\left.2 \frac{\Delta \boldsymbol{c}}{c}\right|_{1 \omega}=\beta \varepsilon_{m}$. The ${ }^{721}$ parameter $\beta$ is found much larger in the granite sample than in the sandstone, as 722 indicated by the larger slope observed for granite in Fig. 13.15. 
Fig. 13.16 Strain dependence for the offset component $\Delta \mathbf{c} /\left.\mathbf{c}\right|_{\mathbf{0} \omega}$ extracted from the Fourier analysis (black curves in Fig. 13.14) for two rock samples of Berea sandstone and Berkeley blue granite. This offset component corresponds to transient elastic softening that reaches about $0.5 \%$ at the maximum strain amplitude. Note the overall transition from quadratic to linear dependence as strain increases from $10^{-7}$ to $10^{-5}$

Fig. 13.17 Strain dependence for the slope component $\boldsymbol{\Delta c} /\left.\boldsymbol{c}\right|_{\mathbf{1} \omega}$ of Berea sandstone and Berkeley blue granite. Note the larger $\boldsymbol{\beta}$ value for the granite sample, as indicated by the larger slopes in Fig. 13.14
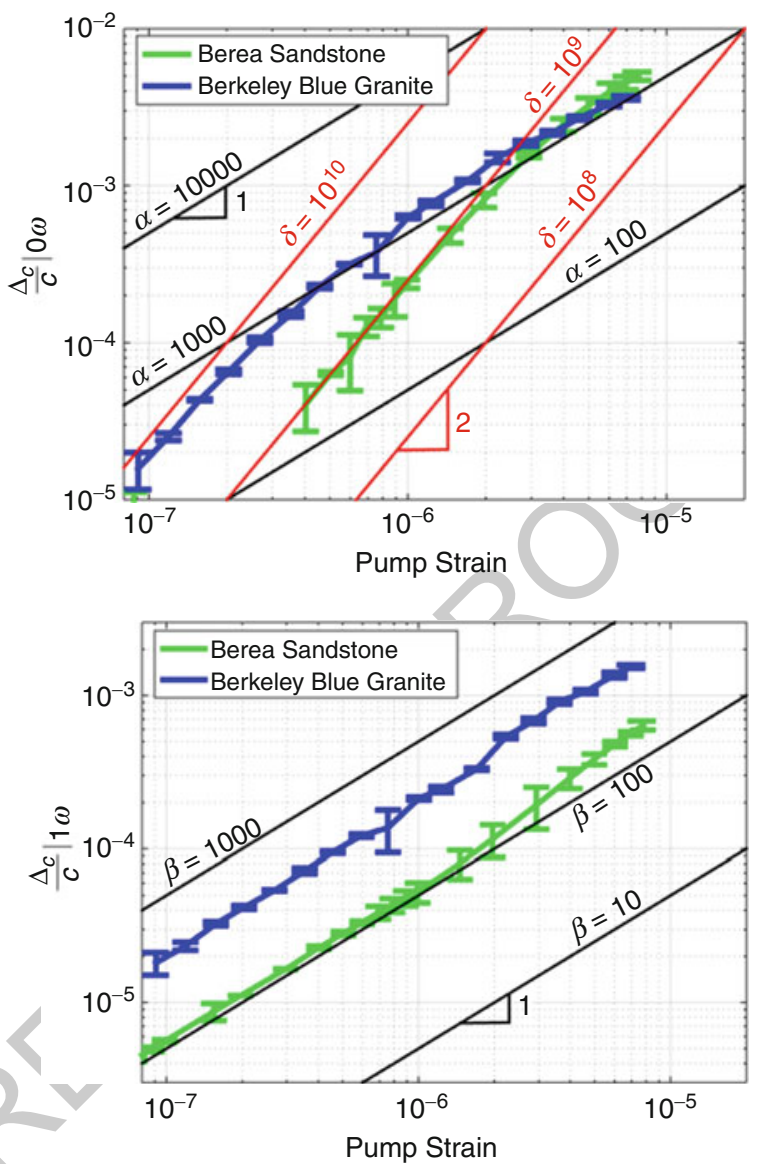

Figure 13.18 shows the $\Delta c /\left.c\right|_{2 \omega}$-component as a function of pump strain 724 amplitude, related to the curvature shape in Fig. 13.15. As for the offset component, 725 we observe a scaling change over the strain range considered [45]. It transitions 726 from quadratic to roughly linear for the Berea sandstone, while the Berkeley blue 727 granite is roughly linear at low strain and lower than 1 at large strain. The quadratic 728 dependence at low strain for Berea can be fitted with $\delta$ using $\left.\frac{\Delta \boldsymbol{M}}{M}\right|_{2 \omega}=\left.2 \frac{\Delta \boldsymbol{c}}{c}\right|_{2 \omega}=729$ $\frac{\delta \varepsilon_{m}^{2}}{2}$. The parameter $\delta$ estimated from either the offset in Fig. 13.16 or the curvature 730 in Fig. 13.18 leads to a value comprised between $10^{8}$ and $10^{9}$ for Berea sandstone 731 (a larger value is found from the offset estimation).

The scaling changes observed for $\Delta c /\left.c\right|_{0} \omega$ and $\Delta c /\left.c\right|_{2 \omega}$ occur at strains where 733 higher order harmonics (in particular, $\Delta c /\left.c\right|_{\mathbf{4} \omega}$ and $\Delta c /\left.c\right|_{\mathbf{6} \omega}$ ) emerge from noise 734 [28, 34]. Rather than using two models corresponding to two different strain 735 ranges, an alternative approach consists in introducing a critical strain level $\varepsilon_{c} \quad 736$ at which the sample transitions from a quadratic to a linear dependence, using 737 


\section{Author's Proof}

13 Dynamic Acousto-Elastic Testing

Fig. 13.18 Strain

dependence for the curvature component $\boldsymbol{\Delta c} /\left.\boldsymbol{c}\right|_{\mathbf{2} \omega}$ of Berea sandstone and Berkeley blue granite. As in Fig. 13.15 for the offset component, a progressive change in amplitude dependence is observed as strain increases from $10^{-7}$ to $10^{-5}$

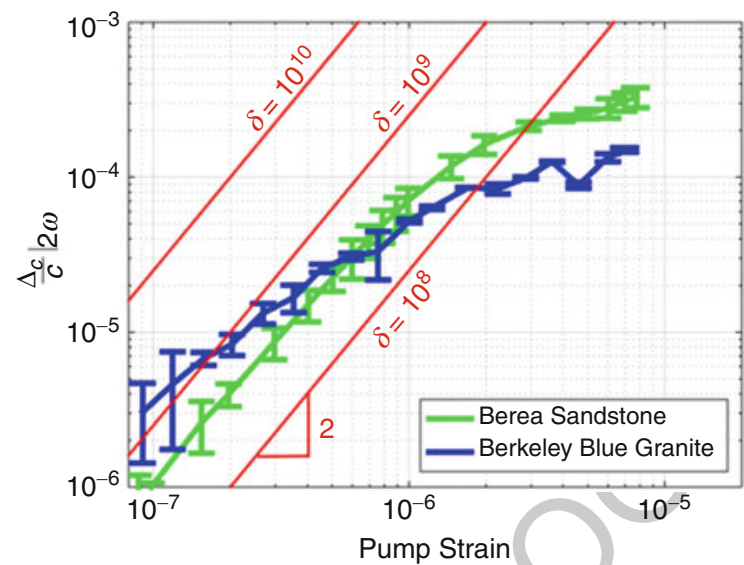

$\left.\frac{\Delta \boldsymbol{M}}{M}\right|_{0 \omega / 2 \omega}=\left.2 \frac{\Delta \boldsymbol{c}}{c}\right|_{0 \omega / 2 \omega}=\Gamma \varepsilon_{c} \tanh \frac{\varepsilon_{m}}{\varepsilon_{c}} \varepsilon_{m}$ (Guyer, personal note). This critical 738 strain, a characteristic of the material, could then also be used to describe the 739 emergence of higher order harmonics.

Finally, a third-more systematic-approach is used in [28] to compare the 741 responses from 6 different rock samples. Ignoring the changes in scaling, each curve 742 in Figs. 13.16, 13.17, and 13.18 is fitted over the whole strain range with $\left.\frac{\Delta c}{c}\right|_{n \omega}=743$ $a \varepsilon_{m}^{v}$. The power-law parameter $v$ is compared for all nonlinear components across 744 samples. A correlation is found between the offset power-law $v_{0 \omega}$, the curvature $v_{2 \omega}, \quad 745$ and the hysteresis area of the loops $\nu_{H}$, whereas the slope component $v_{1 \omega}$ is found 746 independent. This suggests that nonlinearity arises from two main mechanisms. The 747 first one associated with the slope component $\Delta c /\left.c\right|_{1} \omega$ can be referred to as the 748 classical nonlinearity, and as suggested above, is possibly related to opening/closing 749 of cracks and grain contacts. The second one associated with all other components 750 can be referred to as non-classical nonlinearity and is possibly related to shearing 751 mechanisms of grain contacts.

One can ask whether the complex nonlinear effects measured at a few kilohertz still 754 exist at much lower frequency. For instance, do these effects happen when a building 755 vibrates at a few hertz due to the passage of seismic waves?

Both quasi-static tests and resonance-type experiments (see Chap. 2 in this 757 book as well as [51, 59, 60]) have shown that rate effects are inherent to the 758 nonlinear response of poorly cemented materials. For instance, when performing 759 compressional tests on rocks, the hysteresis observed at typical experimental rate 760 $(\sim$ several minutes to an hour) can completely disappear if one performs the same 761 test at a much lower rate ( $\sim$ several hours to a few days) [59]. This observation 762 suggests that the system has enough time to recover from each incremental stress 763 
step when cycled slowly: it remains in its original state and is not brought to a 764 metastable state. On the other hand at larger rates, the initial increase in stress 765 brings the sample to a new state and the subsequent decrease in stress does not 766 follow the same path, leading to hysteresis. Resonance-type tests show similar 767 behaviors when frequency is incrementally increased and decreased around the 768 resonance frequency. The two upward and downward curves do not overlap when 769 the test is performed quite fast, whereas they do overlap when the sweeps are 770 conducted slowly [51]. To investigate such phenomena with DAET and provide 771 further insights on the physical mechanisms at play, acousto-elastic measurements 772 are performed at multiple loading frequencies ranging from quasi-static $(\sim 0.1 \mathrm{~Hz}) \quad 773$ to dynamic $\left(\sim 10^{3}\right)$ regimes [61]. A rod-shaped sample of room-dry Berea sandstone 774 is jacketed and placed upright in a pressure vessel. A large piezoelectric stack is 775 forcing the sample to oscillate uniaxially. A small static overburden stress $(0.5 \mathrm{MPa}) 776$ is previously applied to maintain contact at all phases of the oscillation between the 777 stack and the sample. Two longitudinal transducers operating at $500 \mathrm{kHz}$ are glued 778 on the sides of the sample to monitor the ultrasonic velocity before and during the 779 steady-state oscillations. As for the standard DAET setup described in Fig. 13.4, 780 the probing direction is normal to the loading direction. The Fourier analysis is 781 performed on the nonlinear signatures and amplitudes $\Delta c /\left.c\right|_{n \boldsymbol{\omega}}$ are reported in Fig. 782 13.19 for frequencies spanning three orders of magnitude, constant strain amplitude 783 $\left(\varepsilon=1.4 \times 10^{-5}\right)$, and constant confining pressure $(1 \mathrm{MPa})$. Interestingly, the 784 clustering found when studying multiple rocks at various strain amplitudes applies 785 here too. Indeed, all nonlinear components but the slope $\left(\Delta c /\left.c\right|_{\mathbf{1} \omega}\right)$ increase with 786 frequency, suggesting that the mechanism related to $\Delta c /\left.c\right|_{\mathbf{1} \omega}$ is rather frequency 787 independent (for instance, opening/closing of cracks). On the other hand, the 788 frequency dependence observed for other components reinforces the assumption 789 of friction/adhesion processes at crack interfaces and/or grain boundaries [62, 63]. 790

The increase in nonlinearity with frequency corroborates former studies based on 791 quasi-static and resonance tests [51,59], that is, when dynamic tests are performed 792 slowly enough, the specimen can continuously recover from the changes in loading 793 conditions. Interestingly, the nonlinear components shown in Fig. 13.19 increase 794 only by a factor 2 or 3 over 3 orders of magnitude in frequency, indicating that 795 observations made at the laboratory scale in the kilohertz range are relevant to 796 interpret larger scale observations (civil structures, geotechnical engineering, and 797 seismology).

The term "slow dynamics" was first used by [64] to describe the progressive 800 recovery of the resonance frequency of rocks subsequent to acoustic straining or 801 thermal shocking. Dynamic perturbations of sufficiently high amplitude (strain 802 $>10^{-6}$ ) bring mesoscopic nonlinear materials such as rocks and cementitious 803 materials [50] to a temporary metastable state that manifests itself by a sudden 804 elastic softening (Phase II in Fig. 13.14). Once the perturbation is terminated, 805 the elastic modulus slowly relaxes back towards its unperturbed equilibrium state 806 (Phase VI in Fig. 13.14). This gradual transition of state is termed slow dynamics. $\quad 807$ 


\section{Author's Proof}

13 Dynamic Acousto-Elastic Testing

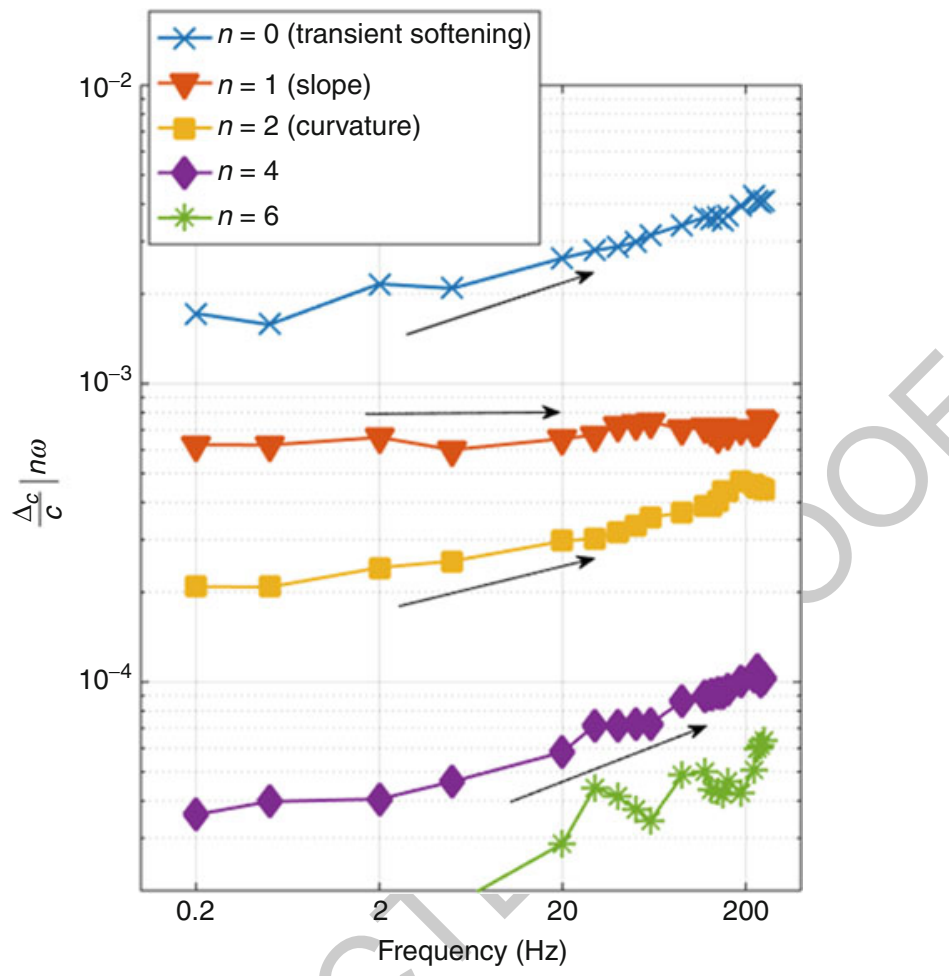

Fig. 13.19 Frequency dependence of the nonlinear components for a sample of Berea sandstone. All nonlinear components increase by a factor 2 or 3 over 3 orders of magnitude increase in frequency, except $\boldsymbol{\Delta} \boldsymbol{c} /\left.\boldsymbol{c}\right|_{\mathbf{1} \omega}$ (slope component) which is frequency independent. Note that these curves are obtained for a constant dynamic strain amplitude $\left(\varepsilon_{m}=1.4 \times 10^{-5}\right)$ and constant confining pressure $(1 \mathrm{MPa})$. Similar observations are made at other confining pressures and oscillation amplitudes [61]

\section{Early Time Vs. Late-Time Recovery}

Earlier empirical observations have unanimously reported a time-logarithmic recov- 809 ery at times $\mathrm{t}>10 \mathrm{~s}$ after terminating the high-amplitude perturbation. The observed 810 behavior appears to be independent of the test material or method used: resonance 811 frequency in disparate rocks and concrete [64], Larsen frequency in cement paste 812 and sandstone [65], and change of velocity in concrete [66]. Consequently, the 813 handful of phenomenological models that have been developed to describe the 814 post-perturbation recovery predict a $\log (t)$ behavior [67]. Despite the universal 815 consensus about the time-logarithmic behavior, there is an experimental evidence 816 for non-logarithmic recovery at earlier times, i.e., $t<10$ s. For example, [65] have 817 measured faster than $\log (t)$ relaxation in cement paste and sandstone at $t \sim 10^{-3} .818$ The recent model proposed by Snieder et al. [68] describes a multi-scale relaxation 819 phenomenon that takes place on different temporal and spatial scales. 


\section{Author's Proof}

S. Haupert et al.
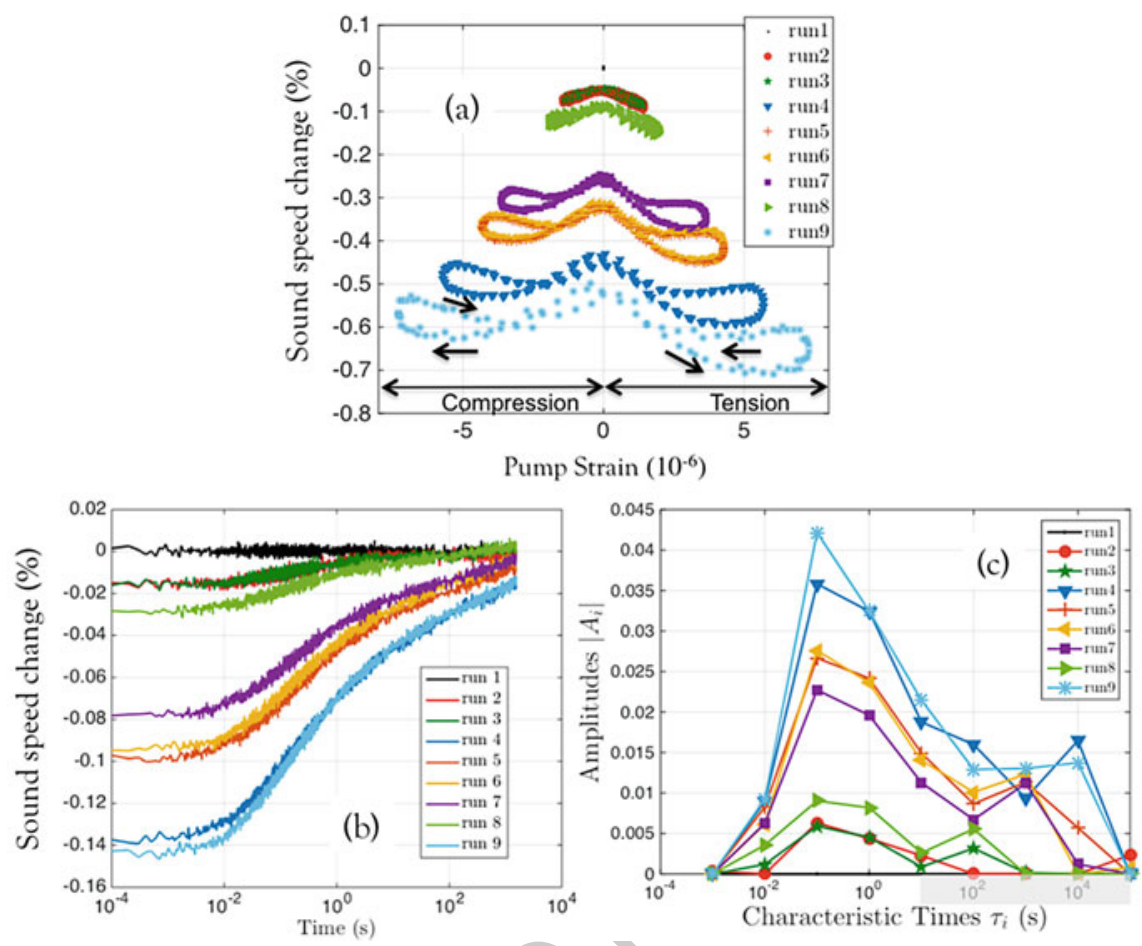

Fig. 13.20 DAET results on a sample of Berea sandstone. Runs 1-9 correspond to different pump strains: (a) Relative change in sound speed vs. pump strain during the non-equilibrium steady-state phase (Phase IV); (b) Progressive recovery of sound speed in Phase VI; (c) The corresponding relaxation spectra show a non-logarithmic recovery behavior at early times $(t<10 \mathrm{~s})$. At later times, the spectrum is rather flat; the recovery is time-logarithmic, as found in previous studies [64]

DAET provides an unprecedented opportunity to investigate the recovery of 821 mesoscopic nonlinear materials at times as early as $t \sim 10^{-4}$ s. Here, we present 822 the results from a series of DAET experiments on a sample of Berea sandstone 823 (Shokouhi et al. in preparation, to appear in 2017). The test apparatus, configuration, 824 and parameters are very similar to those presented in [28] except that the sound 825 speed is probed for a much longer time (about $30 \mathrm{~min}$ ) after stopping the pump. 826 Further, the test is conducted under controlled temperature $T\left({ }^{\circ} \mathrm{C}\right)=23.0 \pm 0.2827$ and relative humidity $\mathrm{RH}(\%)=50.0 \pm 0.2$ conditions. Figure 13.20 presents the 828 obtained results; the sound speed variations with pump strain at steady state (Fig. 829 13.20a) and the corresponding recoveries (Fig. 13.20b).

An examination of the recovery behavior suggests that the material recovers 831 faster at $t>10^{-2} \mathrm{~s}$ than at later times $t>10 \mathrm{~s}$, after which the recovery appears to 832 progress time-logarithmically. For a more quantitative representation, the observed 833 recoveries are described in the form of an exponential series $\sum_{i=1}^{9} A_{i} e^{-t / \tau_{i}}$, where 834 relaxation times $\tau_{i}$ are equally distributed (in a logarithmic way) over the entire 835 


\section{Author's Proof}

13 Dynamic Acousto-Elastic Testing

time range. We find that a summation of nine exponentials is sufficient to fit 836 the data without over fitting. The contribution of each exponential term to the 837 recovery $\left(A_{i}\right)$ or the "recovery spectrum" is shown in Fig. 13.20c. The recovery 838 spectrum is a plot of exponential amplitudes $A_{i}$ vs. the corresponding relaxation 839 times $\tau_{i}$.If wave-speeds were recovering time-logarithmically (i.e., linearly with 840 $\left.\log _{10}(t)\right)$, the spectrum would be flat as was previously observed with resonance- 841 based studies [64]. Unlike these studies, where later time recoveries were probed 842 ( $\tau \geq 10 \mathrm{~s}$ ), the early time recovery $(\tau<10 \mathrm{~s})$ demonstrates preferential recovery time 843 characteristics; the dominant recovery time for the sample is in the order of $10^{-1} \mathrm{~s} .844$ Interestingly, the relaxation spectra for $(\tau \geq 10 \mathrm{~s})$ are almost flat, in close agreement 845 with the earlier observations. Finally, the level of pump strain does not seem to alter 846 the overall shape of the relaxation spectra; they all show a dominant recovery time 847 at about $10^{-1} \mathrm{~s}$. This latter observation suggests a link between the preferential 848 recovery time and the rock microstructure. In fact, our recent observations indicate 849 that the shape of the recovery spectrum is invariant to the changes in relative 850 humidity of the test medium. While increasing the relative humidity increases the 851 nonlinearity, it does not affect the multi-scale recovery rates. This latter observation 852 provides additional evidence for the association of the recovery and microstructure. 853 Furthermore, damage-induced microstructural changes have shown to alter the 854 materials slow dynamics behavior. Earlier studies have shown that damage slows 855 down the late-time recovery of cementitious materials. For example, TenCate et 856 al. [64] show that damaged concrete recovers much slower than intact concrete. 857 Tremblay et al. [66] made similar observations when comparing the late-time 858 recoveries of an intact vs. a stress-damaged concrete sample. The study by Kodjo et 859 al. [69] suggests that the rate of recovery may be used to differentiate two damage 860 processes in concrete.

We use DAET to investigate the influence of damage on early and late-time ${ }_{862}$ recoveries (Shokouhi et al., Ultrasonics, 2017, in press). Figure 13.21 compares the 863 recoveries for two concrete samples: one intact and the other one damaged. Damage 864 is induced by compressing one specimen to about $70 \%$ of its strength. The two 865 specimens are from the same concrete mixture and visually indistinguishable (no 866 visible surface cracking). Ultrasonic wave velocities measured (using compressional 867 wave transducers of center frequency of $150 \mathrm{kHz}$ ) at two different locations across 868 the samples are very similar. The damaged specimen has even a slightly higher linear 869 dynamic modulus than the intact one: $E_{\text {intact }}=33.1 \mathrm{GPa}$ and $E_{\text {damaged }}=33.3 \mathrm{GPa} . \quad 870$ Those moduli are measured by resonance ultrasonic spectroscopy (RUS) testing in 871 the $\mathrm{kHz}$ range [70]. Despite their similar linear acoustic properties, the two samples 872 differ significantly in terms of nonlinear signatures (Fig. 13.21a). The relaxation 873 spectra in Fig. 13.21c show that the substantial recovery of the intact sample takes 874 place for $t<1 \mathrm{~s}$, whereas the damaged sample is still recovering even after $1 \mathrm{~s}$. This 875 observation suggests that damage-induced microstructural changes alter both fast 876 and slow dynamics behaviors and that both signatures can be used to infer the state 877 of damage in materials. 


\section{Author's Proof}

S. Haupert et al.
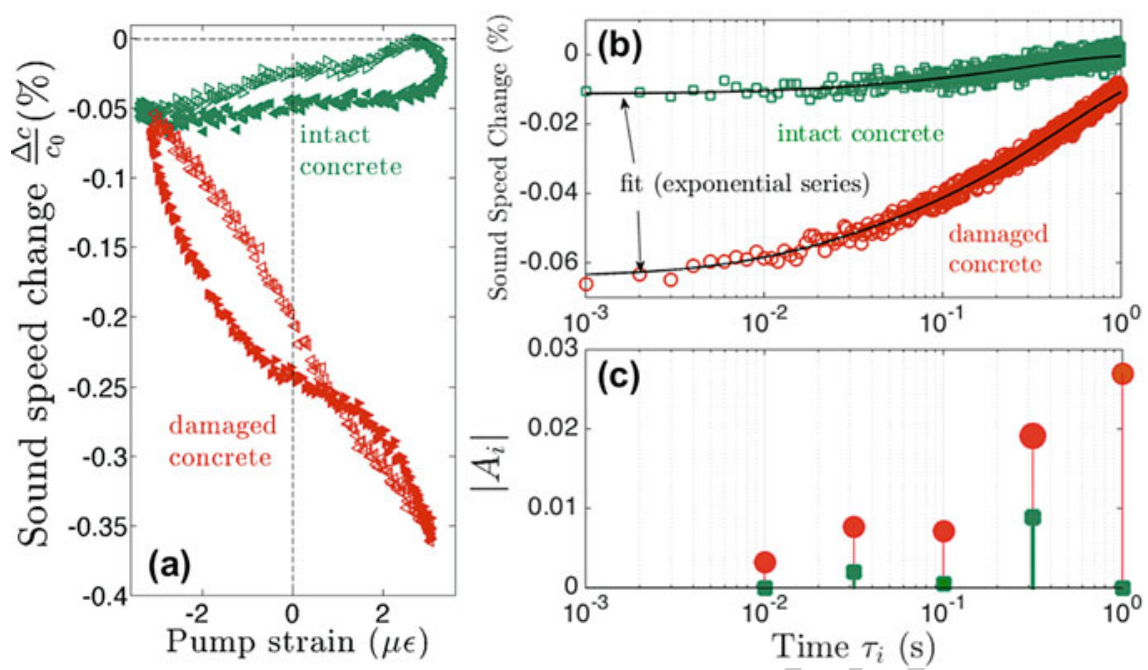

Fig. 13.21 Influence of damage on the DAET response of two concrete samples. (a) Fast dynamics response of intact and damaged concrete samples (phase IV). (b) Slow dynamics response (phase VI). (c) Corresponding relaxation spectra. Less than a second is needed for the intact sample to recover, whereas the damaged sample continues to recover after $1 \mathrm{~s}$

\subsection{Conclusions}

The objective of Dynamic Acousto-Elastic Testing (DAET) is the measurement of 880 the dynamic stress (or strain) dependence of the elasticity of a material at the micro- 881 strain level. In a typical DAET experiment, two elastic waves are simultaneously 882 generated in a material, a low-frequency pump wave and a sequence of identical 883 high-frequency probe waves. The sequence of probe waves determines the local 884 changes of wave-speed induced by the pump wave. These changes can be related to 885 local changes of elasticity of the material. Unlike methods like nonlinear resonant 886 ultrasound spectroscopy and nonlinear wave mixing, DAET reveals the details 887 of the nonlinear elastic behavior over a single wave cycle, including hysteresis 888 and expansion-compression asymmetry. While non-bubbly fluids or homogeneous 889 undamaged solids exhibit a weak and simple dynamic acousto-elastic response, 890 bubbly liquids and damaged or granular solids show large and complicated dynamic 891 acousto-elastic responses. Thus DAET can be a useful technique for nondestructive 892 evaluation of materials. 


\section{Author's Proof}

13 Dynamic Acousto-Elastic Testing

\section{References}

1. P.W. Bridgman, Water, in the liquid and five solid forms, under pressure. Proc. Am. Acad. Arts 895 Sci. 47, 441-558 (1912)

2. F. Birch, The effect of pressure on the modulus of rigidity of several metals and glasses. J. 897 Appl. Phys. 8, 129-133 (1937)

3. P. Van't Klooster, N.J. Trappeniers, S.N. Biswas, Effect of pressure on the elastic constants of 899 noble metals from -196 to $+25^{\circ} \mathrm{C}$ and up to 2500 bar. Physica B 97(1), 65-75 (1979) 900

4. J.C. Swanson, Pressure coefficients of acoustic velocity for nine organic liquids. J. Chem. Phys. 901 2, 689-693 (1934)

5. J.R. Pellam, J.K. Galt, Ultrasonic propagation in liquids: I. Application of pulse technique 903 to velocity and absorption measurements at 15 Megacycles. J. Chem. Phys. 14(10), 608-614 904 (1946)

6. D. Lazarus, The variation of the adiabatic elastic constants of $\mathrm{KCl}, \mathrm{NaCl}, \mathrm{CuZn}, \mathrm{Cu}$, and $\mathrm{Al} 906$ with pressure to 10,000 Bars. Phys. Rev. 76(4), 545-553 (1949) 907

7. A.N. Norris, Nonlinear Acoustics (Academic, New York, 1998), pp. 263-277 908

8. D.S. Hughes, J.L. Kelly, Second-order elastic deformation of solids. Phys. Rev. 92(5), 1145- 909 1149 (1953)

9. T. Bateman, W.P. Mason, H.J. McSkimin, Third-order elastic moduli of germanium. J. Appl. 911 Phys. 32(5), 928-936 (1961)

10. P.B. Nagy, Fatigue damage assessment by nonlinear materials characterization. Ultrasonics 36, 913 375-381 (1998)

11. J.R. Asay, D.L. Lamberson, A.H. Guenther, Pressure and temperature dependence of the 915 acoustic velocities in polymethylmethacrylate. J. Appl. Phys. 40(4), 1768-1783 (1969) 916

12. K.W. Winkler, L. McGowan, Nonlinear acoustoelastic constants of dry and saturated rocks. J. 917 Geophys. Res. 109, B10204 (2004) 918

13. S. Haussühl, W. Chmielewski, Third-order elastic constants of orthorhombic calcium formate. 919 Acta Crystallogr. Sect. A 37(3), 361-364 (1981) 920

14. J.K. Krüger, C. Grammes, K. Stockem, R. Zietz, M. Dettenmaier, Nonlinear elastic properties 921 of solid polymers as revealed by Brillouin spectroscopy. Colloid Polym. Sci. 269(8), 764-771 922 (1991)

15. G. Gremaud, M. Bujard, W. Benoit, The coupling technique: a two-wave acoustic method for 924 the study of dislocation dynamics. J. Appl. Phys. 61(5), 1795-1805 (1987) 925

16. X. Jacob, C. Christophe Barriere, D. Royer, Acoustic nonlinearity parameter measurements in 926 solids using the collinear mixing of elastic waves. Appl. Phys. Lett. 82(6), 886-888 (2003) 927

17. A. Zeiger, K. Jassby, Measurement of acoustoelastic coefficients of Rayleigh waves in steel 928 alloys. J. Nondestruct. Eval. 3(2), 115-124 (1982) 929

18. R. Ellwood, T. Stratoudaki, S.D. Sharples, M. Clark, M.G. Somekh, Determination of the 930 acoustoelastic coefficient for surface acoustic waves using dynamic acoustoelastography: an 931 alternative to static strain. J. Acoust. Soc. Am. 135(3), 1064-1070 (2014) 932

19. G. Renaud, S. Callé, J.-P. Remenieras, M. Defontaine, Exploration of trabecular bone nonlinear 933 elasticity using time-of-flight modulation. IEEE Trans. UFFC 55(7), 1497-1507 (2008) 934

20. G. Renaud, S. Callé, M. Defontaine, Remote dynamic acoustoelastic testing: elastic and 935 dissipative acoustic nonlinearities measured under hydrostatic tension and compression. Appl. 936 Phys. Lett. 94, 11905 (2009)

21. P.J. Westervelt, Scattering of sound by sound. J. Acoust. Soc. Am. 29(2), 199-203 (1957) 938

22. L.H. Taylor, F.R.J. Rollins, Ultrasonic study of three-phonon interactions. I. Theory. Phys. Rev. 939 136(3A), A59-A596 (1964)

23. H. Moreschi, S. Callé, S. Guerard, D. Mitton, G. Renaud, M. Defontaine, Monitoring trabecular 941 bone microdamage using a dynamic acousto-elastic testing method. Proc. Inst. Mech. Eng. 942 225(3), 282-295 (2010)

24. G. Renaud, M. Defontaine, S. Callé, Dynamic acoustoelastic testing of weakly pre-loaded 944 unconsolidated water-saturated glass beads. J. Acoust. Soc. Am. 128(6), 1-11 (2010) 
25. C. Trarieux, S. Callé, H. Moreschi, G. Renaud, M. Defontaine, Modeling nonlinear viscoelas- 946 ticity in dynamic acoustoelasticity. Appl. Phys. Lett. 105(26), 264103 (2014) 947

26. G. Renaud, J.G. Bosch, A.F. van der Steen, N. de Jong, Dynamic acousto-elastic testing applied 948 to a highly dispersive medium and evidence of shell buckling of lipid-coated gas microbubbles. 949 J. Acoust. Soc. Am. 138(5), 2668-2677 (2015)

27. G. Renaud, M. Talmant, S. Callé, M. Defontaine, P. Laugier, Nonlinear elastodynamics in 951 micro-inhomogeneous solids observed by head-wave based dynamic acoustoelastic testing. J. 952 Acoust. Soc. Am. 130(6), 3583-3589 (2011)

28. J. Rivière, P. Shokouhi, R.A. Guyer, P.A. Johnson, A set of measures for the systematic 954 classification of the nonlinear elastic behavior of disparate rocks. J. Geophys. Res. Solid Earth 955 120(3), 1587-1604 (2015)

29. D. Bui, S.A. Kodjo, P. Rivard, B. Fournier, Evaluation of concrete distributed cracks by 957 ultrasonic travel time shift under an external mechanical perturbation: study of indirect and 958 semi-direct transmission configurations. J. Nondestruct. Eval. 32(1), 25-36 (2013) 959

30. Q.A. Vu, V. Garnier, J.F. Chaix, C. Payan, M. Lott, J.N. Eiras, Concrete cover characterisation 960 using dynamic acousto-elastic testing and Rayleigh waves. Constr. Build. Mater. 114, 87-97 961 (2016)

31. J. Rivière et al., Dynamic acousto-elasticity in a fatigue-cracked sample. J. Nondestruct. Eval. 963 33(2), 216-225 (2014)

32. S. Haupert, J. Rivière, B. Anderson, Y. Ohara, T.J. Ulrich, P. Johnson, Optimized dynamic 965 acousto-elasticity applied to fatigue damage and stress corrosion cracking. J. Nondestruct. 966 Eval. 33(2), 226-238 (2014)

33. M. Scalerandi, A.S. Gliozzi, S. Haupert, G. Renaud, M. Ait Ouarabi, F. Boubenider, Investi- 968 gation of the validity of dynamic acoustoelastic testing for measuring nonlinear elasticity. J. 969 Appl. Phys. 118(12), 124905 (2015)

34. J. Rivière, G. Renaud, R.A. Guyer, P.A. Johnson, Pump and probe waves in dynamic acousto- 971 elasticity: comprehensive description and comparison with nonlinear elastic theories. J. Appl. 972 Phys. 114(5), 54905 (2013)

35. T. Gallot, A. Malcolm, T.L. Szabo, S. Brown, D. Burns, M. Fehler, Characterizing the nonlinear 974 interaction of S- and P-waves in a rock sample. J. Appl. Phys. 117(3), 34902 (2015) 975

36. M. Lott et al., Three-dimensional treatment of nonequilibrium dynamics and higher order 976 elasticity. Appl. Phys. Lett. 108(14), 141907 (2016) 977

37. G. Renaud, J. Rivière, S. Haupert, P. Laugier, Anisotropy of dynamic acoustoelasticity in 978 limestone, influence of conditioning, and comparison with nonlinear resonance spectroscopy. 979 J. Acoust. Soc. Am. 133(6), 3706-3718 (2013)

38. J.N. Eiras, Q.A. Vu, M. Lott, J. Payá, V. Garnier, C. Payan, Dynamic acousto-elastic test using 981 continuous probe wave and transient vibration to investigate material nonlinearity. Ultrasonics 982 69, 29-37 (2016)

39. B. Hilloulin et al., Monitoring of autogenous crack healing in cementitious materials by the 984 nonlinear modulation of ultrasonic coda waves, 3D microscopy and X-ray microtomography. 985 Constr. Build. Mater. 123, 143-152 (2016)

40. M.A. Ouarabi, F. Boubenider, A.S. Gliozzi, M. Scalerandi, Nonlinear coda wave analysis of 987 hysteretic elastic behavior in strongly scattering media. Phys. Rev. B 94(13), 134103 (2016) 988

41. G. Renaud, M. Talmant, G. Marrelec, Microstrain-level measurement of third-order elastic 989 constants applying dynamic acousto-elastic testing. J. Appl. Phys. 120(13), 135102 (2016) 990

42. M. Lott, M.C. Remillieux, P.-Y. Le Bas, T.J. Ulrich, V. Garnier, C. Payan, From local to global 991 measurements of nonclassical nonlinear elastic effects in geomaterials. J. Acoust. Soc. Am. 992 140(3), EL231-EL235 (2016)

43. G. Renaud, P.-Y. Le Bas, P.A. Johnson, Revealing highly complex elastic nonlinear (anelastic) 994 behavior of Earth materials applying a new probe: dynamic acoustoelastic testing. J. Geophys. 995 Res. 117(B6), B06202 (2012)

44. C. Payan, T.J. Ulrich, P.-Y. Le Bas, T. Saleh, M. Guimaraes, Quantitative linear and nonlinear 997 resonance inspection techniques and analysis for material characterization: Application to 998 concrete thermal damage. J. Acoust. Soc. Am. 136(2), 537-546 (2014) 


\section{Author's Proof}

13 Dynamic Acousto-Elastic Testing

45. G. Renaud et al., In situ characterization of shallow elastic nonlinear parameters with dynamic 1000 acoustoelastic testing. J. Geophys. Res. Solid Earth 119(9), 6907-6923 (2014) 1001

46. J.A. TenCate, A.E. Malcolm, X. Feng, M.C. Fehler, The effect of crack orientation on the 1002 nonlinear interaction of a P wave with an S wave. Geophys. Res. Lett. 43(12), 6146-6152 1003 (2016)

1004

47. I. Céspedes, Y. Huang, J. Ophir, S. Spratt, Methods for estimation of subsample time delays of 1005 digitized echo signals. Ultrason. Imaging 17, 142-171 (1995) 1006

48. I.Y. Solodov, N. Krohn, G. Busse, CAN: an example of nonclassical acoustic nonlinearity in 1007 solids. Ultrasonics 40(1-8), 621-625 (2002) 1008

49. S. Delrue, K. Van Den Abeele, Three-dimensional finite element simulation of closed 1009 delaminations in composite materials. Ultrasonics 52(2), 315-324 (2012) 1010

50. R.A. Guyer, P.A. Johnson, Nonlinear Mesoscopic Elasticity (Wiley, New York, 2009) 1011

51. J.A. TenCate, Slow dynamics of earth materials: an experimental overview. Pure Appl. 1012 Geophys. 168(12), 2211-2219 (2011) 1013

52. P.A. Johnson, X. Jia, Nonlinear dynamics, granular media and dynamic earthquake triggering. 1014 Nat. Lett. 437(6), 871-874 (2005) 1015

53. F. Brenguier, M. Campillo, C. Hadziioannou, N.M. Shapiro, R.M. Nadeau, E. Larose, 1016 Postseismic relaxation along the san andreas fault at parkfield from continuous seismological 1017 observations. Science 321(5895), 1478-1481 (2008) 1018

54. J.E. Elkhoury, E.E. Brodsky, D.C. Agnew, Seismic waves increase permeability. Nature 1019 441(7097), 1135-1138 (2006) 1020

55. D. Pasqualini, K. Heitmann, J.A. TenCate, S. Habib, D. Higdon, P.A. Johnson, Nonequilibrium 1021 and nonlinear dynamics in Berea and Fontainebleau sandstones: low-strain regime. J. Geophys. 1022 Res. 112, B01204 (2007) 1023

56. K.R. McCall, Theoretical study of nonlinear elastic wave propagation. J. Geophys. Res. Solid 1024 Earth 99(B2), 2591-2600 (1994) 1025

57. K.E.-A. Van Den Abeele, P.A. Johnson, A. Sutin, Nonlinear elastic wave spectroscopy (NEWS) 1026 techniques to discern material damage, part I: nonlinear wave modulation spectroscopy 1027 (NWMS). Res. Nondestruct. Eval. 12, 17-30 (2000) 1028

58. K.R. McCall, R.A. Guyer, Equation of state and wave propagation in hysteretic nonlinear 1029 elastic materials. J. Geophys. Res. 99(12), 23,887-23,897 (1994) 1030

59. K.E. Claytor, J.R. Koby, J.A. TenCate, Limitations of Preisach theory: elastic aftereffect, 1031 congruence, and end point memory. Geophys. Res. Lett. 36(6), L06304 (2009) 1032

60. J.A. TenCate, T.J. Shankland, Slow dynamics in the nonlinear elastic response of Berea 1033 sandstone. Geophys. Res. Lett. 23(21), 3019-3022 (1996) 1034

61. J. Rivière et al., Frequency, pressure, and strain dependence of nonlinear elasticity in Berea 1035 sandstone. Geophys. Res. Lett. 43, 2016GL068061 (2016) 1036

62. V. Gusev, V. Tournat, Amplitude- and frequency-dependent nonlinearities in the presence of 1037 thermally-induced transitions in the Preisach model of acoustic hysteresis. Phys. Rev. B 72, 1038 054104 (2005) 1039

63. C. Pecorari, A constitutive relationship for mechanical hysteresis of sandstone materials. Proc. 1040 R. Soc. Lond. Math. Phys. Eng. Sci. 471(2184), 20150369 (2015) 1041

64. J.A. TenCate, E. Smith, R.A. Guyer, Universal slow dynamics in granular solids. Phys. Rev. 1042 Lett. 85(5), 1020-1023 (2000) 1043

65. O.I. Lobkis, R.L. Weaver, On the Larsen effect to monitor small fast changes in materials. J. 1044 Acoust. Soc. Am. 125(4), 1894-1905 (2009) 1045

66. N. Tremblay, E. Larose, V. Rossetto, Probing slow dynamics of consolidated granular 1046 multicomposite materials by diffuse acoustic wave spectroscopy. J. Acoust. Soc. Am. 127(3), 1047 1239-1243 (2010) 1048

67. O.O. Vakhnenko, V.O. Vakhnenko, T.J. Shankland, J.A. Ten Cate, Strain-induced kinetics of 1049 intergrain defects as the mechanism of slow dynamics in the nonlinear resonant response of 1050 humid sandstone bars. Phys. Rev. E 70(1), 015602 (2004) 1051

68. R. Snieder, C. Sens-Schönfelder, R. Wu, The time dependence of rock healing as a universal 1052 relaxation process, a tutorial. Geophys. J. Int. 208(1), 1-9 (2017) 


\section{Author's Proof}

S. Haupert et al.

69. A.S. Kodjo, P. Rivard, F. Cohen-Tenoudji, J.-L. Gallias, Impact of the alkali-silica reaction 1054 products on slow dynamics behavior of concrete. Cem. Concr. Res. 41(4), 422-428 (2011)

70. T.J. Ulrich, K.R. McCall, R.A. Guyer, Determination of elastic moduli of rock samples using 1056 resonant ultrasound spectroscopy. J. Acoust. Soc. Am. 111(4), 1667-1674 (2002) 